\title{
FAR-INFRARED OBSERVATIONS OF THE VERY LOW LUMINOSITY EMBEDDED SOURCE L1521F-IRS IN THE TAURUS STAR-FORMING REGION
}

\author{
Susan Terebey ${ }^{1}$, Michel Fich ${ }^{2}$, Alberto Noriega-Crespo $^{3}$, Deborah L. Padgett $^{3}$, Misato Fukagawa $^{4}$, Marc Audard $^{5}$, \\ Tim Brooke ${ }^{3}$, Sean Carey ${ }^{3}$, Neal J. Evans II ${ }^{6}$, Manuel Guedel ${ }^{7}$, Dean Hines ${ }^{8}$, Tracy Huard ${ }^{9}$, Gillian R. KNapp ${ }^{10}$, \\ Caer-Eve McCabe ${ }^{3}$, Francois Menard $^{11}$, Jean-Louis Monin $^{11}$, and Luisa Rebull ${ }^{3}$ \\ ${ }^{1}$ Department of Physics and Astronomy PS315, 5151 State University Drive, California State University at Los Angeles, Los Angeles, CA 90032, USA; \\ sterebe@calstatela.edu \\ 2 Department of Physics and Astronomy, University of Waterloo, Waterloo, ON N2L 3G1, Canada; fich@ uwaterloo.ca \\ ${ }^{3}$ Spitzer Science Center, MC 220-6, California Institute of Technology, Pasadena, CA 91125, USA \\ ${ }^{4}$ Japan Aerospace Exploration Agency, Institute of Space and Astronautical Science, Japan \\ ${ }^{5}$ ISDC, Switzerland and Observatoire de Genève, Switzerland \\ ${ }^{6}$ University of Texas, Austin, TX, USA \\ ${ }^{7}$ Paul Scherrer Institute, Switzerland \\ ${ }^{8}$ Space Science Institute, Boulder, CO, USA \\ ${ }^{9}$ University of Maryland, College Park, MD, USA \\ ${ }^{10}$ Princeton University, NJ, USA \\ ${ }^{11}$ LAOG, Grenoble, France \\ Received 2008 June 30; accepted 2009 February 27; published 2009 April 27
}

\begin{abstract}
We investigate the environment of the very low luminosity object L1521F-IRS using data from the Taurus Spitzer Legacy Survey. The MIPS $160 \mu \mathrm{m}$ image shows both extended emission from the Taurus cloud and emission from multiple cold cores over a $1^{\circ} \times 2^{\circ}$ region. Analysis shows that the cloud dust temperature is $14.2 \pm 0.4 \mathrm{~K}$ and the extinction ratio is $A_{160} / A_{K}=0.010 \pm 0.001$ up to $A_{V} \sim 4 \mathrm{mag}$. We find $\kappa_{160}=0.23 \pm 0.046 \mathrm{~cm}^{2} \mathrm{~g}^{-1}$ for the specific opacity of the gas-dust mixture. Therefore, for dust in the Taurus cloud we find that the $160 \mu \mathrm{m}$ opacity is significantly higher than that measured for the diffuse interstellar medium, but not too different from dense cores, even at modest extinction values. Furthermore, the $160 \mu \mathrm{m}$ image shows features that do not appear in the IRAS $100 \mu \mathrm{m}$ image. We identify six regions as cold cores, i.e., colder than $14.2 \mathrm{~K}$, all of which have counterparts in extinction maps or $\mathrm{C}^{18} \mathrm{O}$ maps. Three of the six cores contain embedded young stellar objects, which demonstrates the cores are sites of current star formation. We compare the effects of L1521F-IRS on its natal core and find there is no evidence for dust heating at 160 or $100 \mu \mathrm{m}$ by the embedded source. From the infrared luminosity $L_{\mathrm{TIR}}=0.024 L_{\odot}$ we find $L_{\text {bol_int }}=0.034-0.046 L_{\odot}$, thus confirming the source's low luminosity. Comparison of L1521F-IRS with theoretical simulations for the very early phases of star formation appears to rule out the first core collapse phase. The evolutionary state appears similar to or younger than the class 0 phase, and the estimated mass is likely to be substellar.
\end{abstract}

Key words: circumstellar matter - stars: formation - stars: individual (L1521F-IRS, L1527-IRS,

IRAS 04368+2557) - stars: low-mass, brown dwarfs

\section{INTRODUCTION}

Investigation of starless cloud cores provides a way to study the initial conditions of star formation, where prestellar cores are those judged to be closest to gravitational cloud collapse. The L1521F cloud core in the Taurus molecular cloud at $140 \mathrm{pc}$ distance (Torres et al. (2007), and references therein) is a very dense $\left(n \sim 10^{6} \mathrm{~cm}^{-3}\right)$ and cold $(T \sim 9 K)$ cloud core containing $2-5 M_{\odot}$ of gas detected in $\mathrm{NH}_{3}$ and other dense gas tracers (Codella et al. 1997; Onishi et al. 1999; Crapsi et al. 2004, 2005; Shinnaga et al. 2004).

Onishi et al. (1999) singled out L1521F (their MC 27) as special in mapping surveys of Taurus cores, and argued that the high density and infall asymmetry seen in the $\mathrm{HCO}^{+}(3-2)$ line indicated a core in the earliest stages of gravitational collapse with a free-fall timescale of $10^{3}$ to $10^{4} \mathrm{yr}$. Consistent with the young age, IRAS observations indicated an upper limit of about $0.1 L_{\odot}$ for the luminosity of any possible embedded object. The presence of infall asymmetries in other tracers and an advanced chemical clock led other authors to similar conclusions (Crapsi et al. 2004; Lee et al. 2004; Sohn et al. 2007). Models of the cloud structure were consistent with the picture of a dense cold core heated externally by the interstellar radiation field (Onishi et al. 1999; Crapsi et al. 2004).
The Spitzer Space Telescope (SST; Werner et al. 2004) detected a very low luminosity infrared (IR) source in the $\mathrm{L} 1521 \mathrm{~F}$ prestellar core, a source too faint to appear in the IRAS catalog (Terebey et al. 2005; Bourke et al. 2006). Bourke et al. (2006) present submillimeter and independent Spitzer data for the core. The source is detected at all IRAC and MIPS wavelengths ranging from 3.6 to $70 \mu \mathrm{m}$ in wavelength. In this paper, we further present $160 \mu \mathrm{m}$ data for the cloud. The source's low luminosity suggests the object is substellar, and its similarity to class 0 sources suggests it is extremely young. An object that predates Spitzer, IRAM 04191+1522, is a low luminosity $\sim 0.1 L_{\odot}$ class 0 source in Taurus detected by ISO longward of $60 \mu \mathrm{m}$ (André et al. 1999; Dunham et al. 2006), that is now suggested as a very low luminosity object (VeLLO) (di Francesco et al. 2007; Dunham et al. 2008). However, IRAM $04191+1522$ differs from L1521F by exhibiting both a molecular outflow and centimeter radio emission. Other comparable objects detected by Spitzer include VeLLOs in the L1014 and L328 molecular clouds (Young et al. 2004; Lee, \& c2d Team 2005; Huard et al. 2006). The spectral energy distributions (SED) are similar, steeply rising with strong emission at 24 and $70 \mu \mathrm{m}$ in the IR. Dunham et al. (2008) presented numerous VeLLO candidates from the Spitzer c2d 
survey. The low luminosity of the VeLLOs suggests that their masses are quite small. The presence of dense cores and infall asymmetries toward the better studied sources suggests that some may represent a very early stage of star formation, of accretion onto a substellar mass central object.

Models of early cloud collapse define two distinct phases, the first protostellar core, when gas in the central region first becomes opaque at $T \sim 100 \mathrm{~K}$ and heats up to $T \sim 2000 \mathrm{~K}$ at which point begins the second core phase, when molecular hydrogen dissociates and a hydrostatic object at stellar density forms. The properties of the first core depend sensitively on the initial conditions and assumed physics but are roughly $0.01-0.08 M_{\odot}$ for mass, $10 \mathrm{AU}$ for radius and $L<0.1 L_{\odot}$ for luminosity (Boss \& Yorke 1995; Bate 1998; Saigo et al. 2008). Numerical simulations suggest the model luminosity then shoots up to $1.0 L_{\odot}$ as the second protostellar core undergoes runaway collapse of a $0.001 M_{\odot}$, i.e., Jupiter mass object. The second core continues to accrete mass but other properties, such as luminosity, at times less than $\sim 10,000$ yr are still unknown, until the object becomes older and more massive, and is detectable as a protostar or young brown dwarf. Stamatellos et al. (2005) explored whether this phase would be observable, in the context of collapse models with high infall rates. Prior to collapse, the SED of the model cloud peaks near $190 \mu \mathrm{m}$ due to heating by the interstellar radiation field. They find significant additional heating occurs after the accretion luminosity exceeds 5.0 $L_{\odot}$, producing a peak wavelength as short as $90 \mu \mathrm{m}$ during the class 0 phase. In the context of these models, it appears VeLLOs are unlikely to modify the submillimeter SED of the cloud. Young et al. (2004) reach a similar conclusion for the L1014 VeLLO source. Far-IR wavelengths are also problematic: previous work by Boulanger \& Perault (1988) using IRAS data found that the externally heated molecular cloud contributes a significant amount of $0.6 L_{\odot} / M_{\odot}$ to the IR luminosity.

To better understand the properties of L1521F-IRS and its relation to early phases of star formation we analyze the MIPS $160 \mu \mathrm{m}$ and IRAS $100 \mu \mathrm{m}$ data near the source. The molecular cloud appears to dominate the emission at these wavelengths, and our goal is to better define the peak of the SED by determining the contribution of the embedded source to the flux. Using these data we also determine the properties of the $\mathrm{L} 1521 \mathrm{~F}$ cloud core at $160 \mu \mathrm{m}$ and relate it to other $160 \mu \mathrm{m}$ cores in the region.

\section{OBSERVATIONS}

\subsection{Spitzer Observations}

The Spitzer data near L1521F derive from the Taurus Spitzer Legacy Survey (D. L. Padgett et al. 2009, in preparation), a large $44 \mathrm{deg}^{2}$ map of the Taurus star-forming region at seven wavelengths ranging from 3.6 to $160 \mu \mathrm{m}$. The nominal spatial resolution of Spitzer is about 1.7 at 3.6, 4.5, 5.8, and $8.0 \mu \mathrm{m}$ with the IRAC instrument, and is 6,18 , and $40^{\prime \prime}$ at 24,70 , and $160 \mu \mathrm{m}$ with the MIPS instrument (Fazio et al. 2004; Rieke et al. 2004). The region near L1521F was observed on 2005 February 23 by IRAC and 2005 March 2 by MIPS. See D. L. Padgett et al. (2009, in preparation) for an overview of the survey parameters and data processing.

The $160 \mu \mathrm{m}$ data obtained in fast scan mode mapping does not have enough redundancy to fill in completely all the gaps due to a dead readout and the intermediate gap between the array detector rows (MIPS Data Handbook ver. 3.32007 August 24). Furthermore with only five pointings per pixel, the effects of
Table 1

L1521F-IRS Source Parameters

\begin{tabular}{lcccc}
\hline \hline Name & $D$ & $L_{\mathrm{TIR}}$ & $\begin{array}{c}\text { R.A. } \\
(\mathrm{J} 2000)\end{array}$ & $\begin{array}{c}\text { Decl. } \\
(\mathrm{J} 2000)\end{array}$ \\
\hline L1521F-IRS 1 & 140 & 0.02 & $04: 28: 38.92$ & $+26: 51: 36.2$ \\
\hline
\end{tabular}

Notes. IR luminosity from Table 5 and as defined in the text. Positions at other wavelengths are the same as the $24 \mu \mathrm{m}$ position, within measurement uncertainties. Position uncertainty at $3.6,4.5$, and $5.8 \mu \mathrm{m}$ is $1^{\prime \prime} .4$, and at $8.0 \mu \mathrm{m}$ is $1^{\prime \prime} .8$.

Table 2

Aperture-Corrected Photometry of L1521F IRS

\begin{tabular}{lcccc}
\hline \hline Telescope & $\begin{array}{c}\lambda \\
(\mu \mathrm{m})\end{array}$ & $\begin{array}{c}\text { PSF }^{\mathrm{a}} \\
\left({ }^{\prime \prime}\right)\end{array}$ & $\begin{array}{c}\text { Flux density }^{\mathrm{b}} \\
(\mathrm{mJy})\end{array}$ & $\begin{array}{c}\sigma \\
(\mathrm{mJy})\end{array}$ \\
\hline Spitzer & 3.6 & 1.66 & $0.20^{\mathrm{c}}$ & 0.04 \\
& 4.5 & 1.72 & $0.35^{\mathrm{c}}$ & 0.07 \\
& 5.8 & 1.88 & $0.48^{\mathrm{c}}$ & 0.10 \\
& 8.0 & 1.98 & $0.98^{\mathrm{c}}$ & 0.2 \\
& 24 & 5.9 & 24 & 1.2 \\
IRAS & 70 & 16 & 460 & 90 \\
& 100 & 118 & $<2000$ & $\ldots$
\end{tabular}

Notes.

a Nominal PSF from Spitzer Observer's Manual (IRAC) or Data Handbook (MIPS).

b IRAC photometry uses radius 2 pixel $=2$ ". 4 aperture and 2-6 pixel sky background. MIPS 24 uses PRF fitting. MIPS 70 uses radius 3 pixel $=12^{\prime \prime}$ aperture.

${ }^{c}$ Source is extended so flux depends on aperture and background.

hard radiation hits and saturation translate into small regions without data. To deal with these gaps and to preserve as much as possible the diffuse emission, a 3 pixel $\times 3$ pixel $\left(15^{\prime \prime}\right.$ pixel $\left.^{-1}\right)$ median filter is applied to the image. The net effect is a slight redistribution of surface brightness $(\sim 15 \%)$ and smearing of the original beam from $40^{\prime \prime}$ to about $1^{\prime}$ in size. To quantify the effect we measured the resulting spatial resolution in two ways. First we determined an FWHM of 52" for a theoretical $160 \mu \mathrm{m}$ PRF that was processed using the same 3 pixel window filter. We note that most objects appear extended in the $160 \mu \mathrm{m}$ mosaic. However we were able to measure an empirical average FWHM of 59" for five pointlike sources, which we take to be the effective spatial resolution of our $160 \mu \mathrm{m}$ image.

Table 1 gives the Spitzer position of the embedded IR source, measured at $24 \mu \mathrm{m}$ to be least affected by extinction and hence most accurately detect the central source. Positions determined at shorter IRAC wavelengths are the same within the measurement uncertainties. The source is extended at IRAC wavelengths, hence the fluxes given in Table 2 depend on both the aperture and background determination. The flux density values are comparable to but differ from those in Bourke et al. (2006). Given that the source is extended we ascribe the difference as due to a difference in the photometric method.

\subsection{IRAS Observations}

Images at 60 and $100 \mu \mathrm{m}$ were obtained ${ }^{12}$ from the HighResolution IRAS Galaxy Atlas (IGA), an atlas that includes the galactic plane, Orion, Taurus-Auriga, and $\rho$ Oph regions (Cao et al. 1997). The coordinates of IGA images outside the galactic plane are given in 1950 equatorial coordinates. In order to

\footnotetext{
12 http://irsa.ipac.caltech.edu/data/IGA/
} 


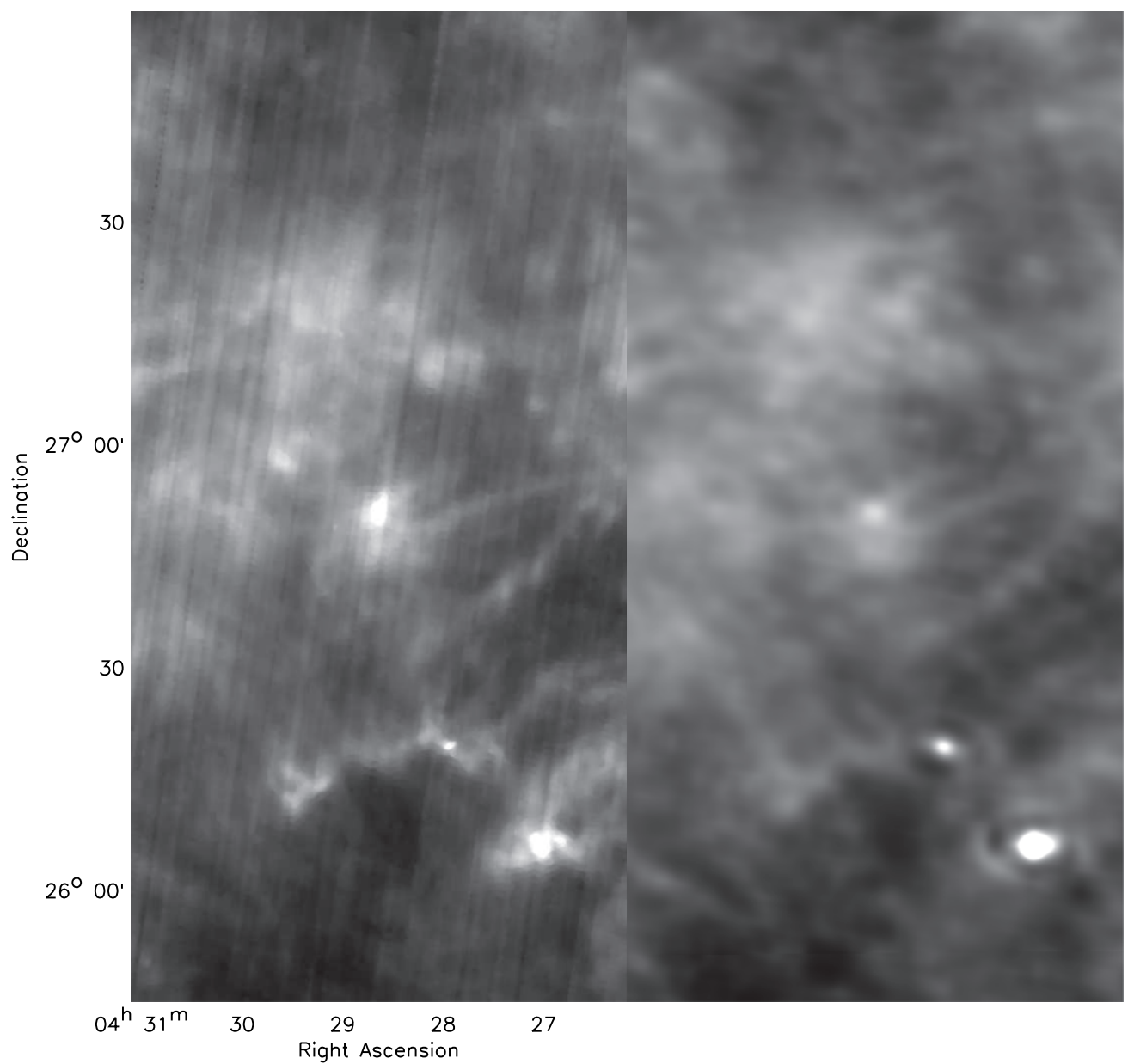

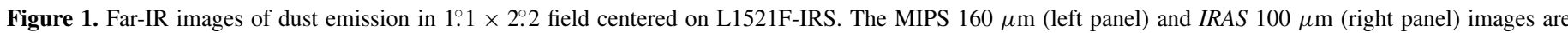

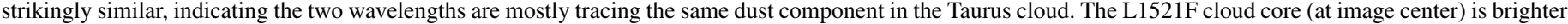

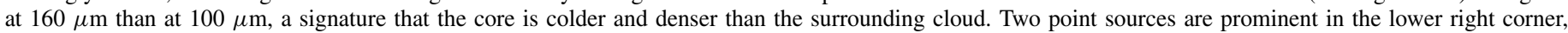
especially at $100 \mu \mathrm{m}$, and indicate regions of higher than average dust temperature. The two bright sources are IRAS $04248+2612$ and DG Tau from E to W.

successfully precess and work with the images, several changes were made to the original FITS headers using the Goddard IDL Astron Library. First, a misspelled keyword RADECSYS was removed and replaced with the keyword RADESYS. Second, the keyword LONPOLE $=0.0$ was added. In addition, the reference pixels CRPIXn and reference positions CRVALn were changed to be at the image centers from their original off-image locations. This last crucial change appeared to minimize differences in various FITS software implementations of the CAR projection type.

The IGA images were generated using the High Resolution (HIRES) resolution enhancement algorithm and therefore have higher spatial resolution $\left(2^{\prime}\right)$ than the native IRAS $(3.8 \times$ $5: 4$ ), ISSA (4!3), or IRIS (Improved Reprocessing of IRAS Survey) (4.3) survey images at $100 \mu \mathrm{m}$ wavelength (Cao et al. 1997; Milville-Deschenes \& Lagache 2005). We constructed an empirical point-spread function (PSF) using several sources in the Taurus survey region to find an effective spatial resolution at $100 \mu \mathrm{m}$ of $140^{\prime \prime} \times 110^{\prime \prime}(\mathrm{FWHM})$, about a factor of 2 times lower than that for the Spitzer MIPS $160 \mu \mathrm{m}$ data.

\section{RESULTS}

\subsection{Morphology of Far-IR Emission}

In Figure 1, far-IR images of dust emission near L1521F-IRS show a striking similarity in the diffuse emission, indicating that the 160 and $100 \mu \mathrm{m}$ wavelengths are mostly tracing the same dust component in the Taurus cloud. The images are centered on the position of L1521F-IRS, but throughout the $1.1 \times 2.2$ field the emission at $160 \mu \mathrm{m}$ is extended with respect to the spatial resolution of $59^{\prime \prime}=8300 \mathrm{AU}=0.04 \mathrm{pc}$ at $140 \mathrm{pc}$ distance.

In the central $5^{\prime}$ the L $1521 \mathrm{~F}$ cloud core is systematically brighter at $160 \mu \mathrm{m}$ than at $100 \mu \mathrm{m}$, a signature that the core is colder and denser than the surrounding cloud. In addition, the young stellar objects (YSOs) IRAS $04248+2612$ and DG Tau appear as prominent point sources in the lower right corner and indicate regions of higher than average dust temperature. The ringing at $100 \mu \mathrm{m}$ around the two point sources is an artifact of the HIRES processing. Other Taurus YSOs fall within the region shown (Figure 1), but only the two named sources show significant emission at these wavelengths.

Previous analyses of the diffuse emission seen by IRAS at 60 and $100 \mu \mathrm{m}$ show that the cirrus can be modeled as emission from dust associated with both the atomic and molecular components of the interstellar medium (ISM) (Terebey \& Fich 1986; Boulanger \& Perault 1988; Wood et al. 1994; Abergel et al. 1994). This extended component is variously referred to as galactic emission, IR cirrus, or the diffuse ISM. Boulanger et al. (1988) modeled the diffuse IRAS cirrus as due to dust heated by the interstellar radiation field and find that the dust is not in thermal equilibrium except at the longest $(100 \mu \mathrm{m})$ wavelength. The morphology of the diffuse emission in the new 


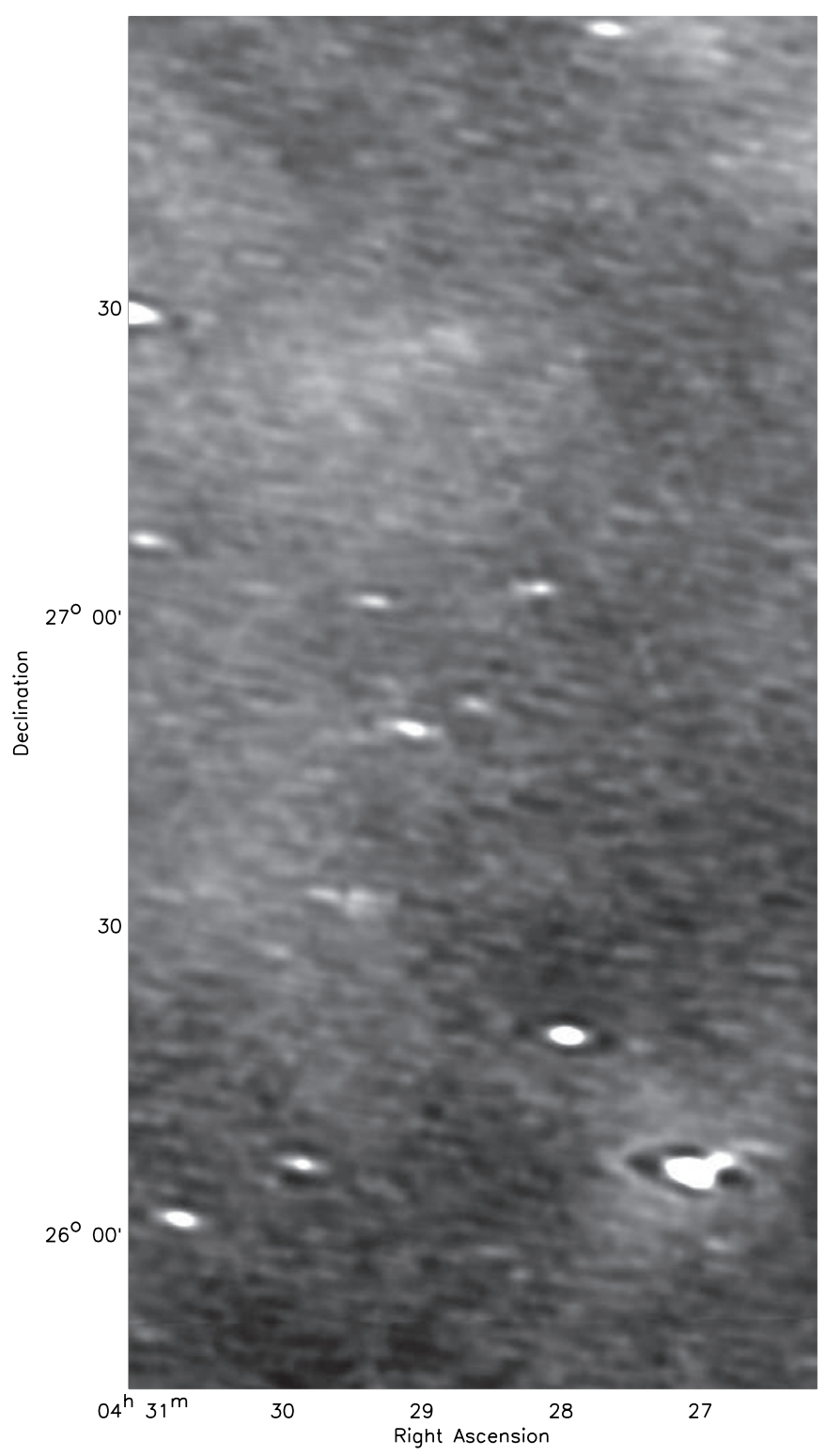

Figure 2. Extended dust emission is also seen across the IRAS $60 \mu \mathrm{m}$ image but there is less correlation with the $160 \mu \mathrm{m}$ image (Figure 1). The $60 \mu \mathrm{m}$ emission appears related but not cospatial, as if tracing warmer dust associated with the cloud surface. Field of view same as Figure 1. A faint but marginally detected source appears at the position of L1521F-IRS, exactly at the image center. Most point sources seen at $60 \mu \mathrm{m}$ do not appear at longer wavelengths.

MIPS $160 \mu \mathrm{m}$ data shows it to be fundamentally the same as the IRAS $100 \mu \mathrm{m}$ emission, albeit viewed at higher spatial resolution.

However, emission at the shorter $60 \mu \mathrm{m}$ wavelength necessarily implies the presence of warmer dust. This has long been understood as a consequence of the Planck function, where emission from cooler dust shifts to the Wien part of the spectral curve and becomes quite faint. Figure 2 shows extended emission along with numerous point sources in a $60 \mu \mathrm{m} I R A S$ image; the $70 \mu \mathrm{m}$ MIPS image (not shown) shows the point sources at higher $\left(\sim 20^{\prime \prime}\right)$ spatial resolution but has less sensitivity to the extended component. The morphology of the $60 \mu \mathrm{m}$ diffuse emission in Figure 2 appears related to the $160 \mu \mathrm{m}$ emission (Figure 1) but not cospatial, as if representing emission from the surface layers of externally irradiated clouds. The embedded source L1521F-IRS appears as a marginally detected pointlike source in the center of the image. However, the object is found in the IRAS Point Source Rejects Catalog (i.e., detected but not reliable) as R04255+2644, which would explain why Onishi et al. (1999) reported the source was not detected by $I R A S$. Apart from the faint point source, there is no extended emission in the central $5^{\prime}$ of the IRAS image (confirmed by the $70 \mu \mathrm{m}$ MIPS image) comparable to that seen at 100 and $160 \mu \mathrm{m}$ (Figure 1) from the cloud core. Our focus in this work is on the dense cold component and we do not further consider the $60 \mu \mathrm{m}$ diffuse emission.

\subsection{Analysis of Extended Dust Emission}

Before determining the temperature and optical depth we first give expressions for the intensity. Assuming dust that is in thermal equilibrium at temperature $T$, then at frequency $v$ the intensity is given by $I_{v}=\left(1-e^{\tau_{v}}\right) B_{v}(T)$, where $\tau_{v}$ is the optical depth and $B_{v}(T)$ is the Planck function. In the limit of small optical depth, adequate for our purpose, the intensity becomes

$$
I_{v} \approx \tau_{v} B_{v}(T)
$$

From this expression we see that taking the ratio of the intensity at two frequencies leads directly to the dust temperature, if the frequency/wavelength-dependent properties of the dust are known. In practice a range of dust temperatures will be present, but at a given frequency a limited range of temperatures contributes significantly to the intensity, as one "loses" cold dust whose emission falls on the Wien part of the blackbody curve. The contribution of small stochastically heated grains can be neglected at these wavelengths; equilibrium thermal emission from big grains (greater than $0.25 \mu \mathrm{m}$ ) dominates the emission at $\lambda \gtrsim 70-80 \mu m$ (Li \& Draine 2001).

The optical depth can be expressed in terms of the specific opacity $\kappa_{v}$ in units of $\mathrm{cm}^{2} \mathrm{~g}^{-1}$, the density of the gas-dust mixture $\rho$, and the path length $L$ by

$$
\begin{aligned}
\tau_{v} & =\kappa_{\nu} \rho L \\
& =\kappa_{\nu} \mu m_{\mathrm{H}} N_{\mathrm{H}}, \\
& =\sigma_{v} N_{\mathrm{H}},
\end{aligned}
$$

and where $\mu=1.38$ is assumed for the mean molecular weight per hydrogen atom, $m_{\mathrm{H}}$ is the mass of a hydrogen atom, $N_{\mathrm{H}}$ is the hydrogen column density, and $\sigma_{v}$ is the opacity in units of $\mathrm{cm}^{2}$ per $\mathrm{H}$ atom. The frequency dependence of the opacity is often expressed in terms of the power-law index $\beta$ where $\kappa_{v}=\kappa_{o}\left(\nu / \nu_{o}\right)^{\beta}$ at some fiducial frequency $\nu_{o}$, and where $\beta$ ranges from roughly 1.5 to 2 at IR wavelengths. Based on their definitions, the relation between extinction and optical depth is given by $A_{\lambda}=2.5 \log (e) \tau_{\lambda}=1.0857 \tau_{\lambda}$ and moreover the extinction opacity $\kappa_{v}=\kappa_{v s c a}+\kappa_{v a b s}$ includes both scattering and absorption. The scattering efficiency is specified by the albedo; at long wavelengths the albedo is zero, hence $\kappa_{v}=$ $\kappa_{\text {vabs }}$ for no scattering, which is the regime that Equation (1) describes.

\subsection{Dust Temperature}

Figure 3 shows that there is a strong linear correlation between the 160 and $100 \mu \mathrm{m}$ images. This indicates that the emission can be well modeled using a single dust temperature but varying optical depth. The two regions immediately surrounding the sources IRAS 04248+2612 and DG Tau depart from the linear trend, which suggests there is internal heating by these YSOs. 


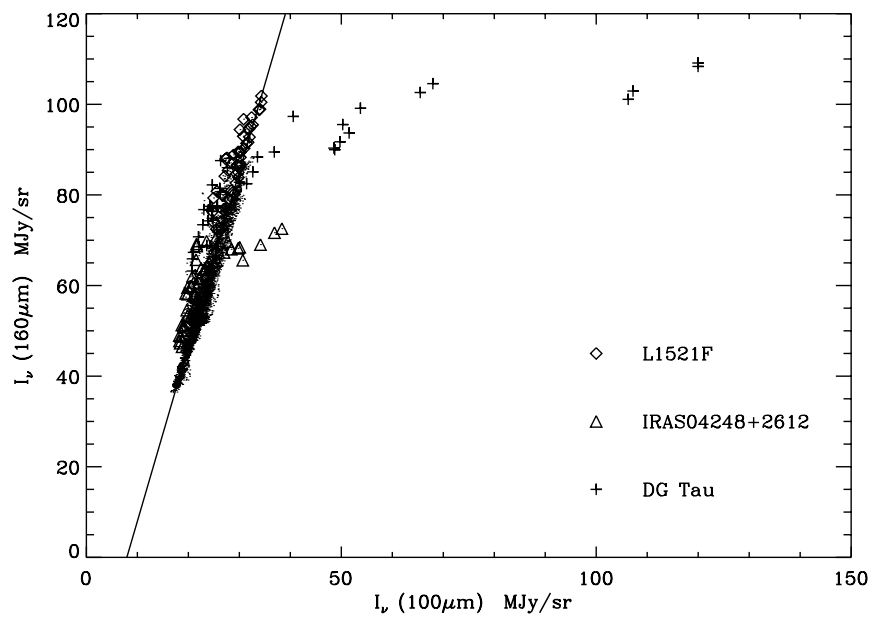

Figure 3. There is a strong linear correlation between the resolution matched MIPS $160 \mu \mathrm{m}$ and IRAS $100 \mu \mathrm{m}$ emission. Solid line shows a linear fit to the data while symbols show regions surrounding specific objects. Excess $100 \mu \mathrm{m}$ emission indicates warm dust around the YSOs IRAS04248+2612 and DG Tau. No evidence of warm dust is seen toward L1521F-IRS, suggesting the source luminosity is too low to provide significant dust heating.

Table 3

Taurus Cloud Dust Temperature Based on 160 and $100 \mu \mathrm{m}$ Data

\begin{tabular}{lcccccc}
\hline \hline$C_{0}$ & $\sigma_{0}$ & $C_{1}$ & $\sigma_{1}$ & $\beta$ & $C_{1 \text { corr }}{ }^{\mathrm{a}}$ & $\begin{array}{c}T_{\text {dust }} \\
(\mathrm{K})\end{array}$ \\
\hline 7.94 & 0.12 & 0.259 & 0.002 & 2.0 & 0.235 & 14.2 \\
& & & & 1.5 & 0.235 & 15.1 \\
\hline
\end{tabular}

Notes. Coefficients and uncertainty of linear fit to data $\mathrm{I} 100=\mathrm{C} 0+\mathrm{C} 1 * \mathrm{I} 160$ shown in Figure 3.

a Corrected slope after applying MIPS and IRAS color corrections based on Equation (1).

Table 3 gives the results of a linear fit to the data, after masking out the warm dust in a $6.5 \times 6.5$ square region near the two named YSOs. In addition, the $160 \mu \mathrm{m}$ image was smoothed to the approximate $100 \mu \mathrm{m}$ resolution via convolution by the empirical IRAS $100 \mu \mathrm{m}$ PSF, and the resulting images sampled at 7 pixels $=56^{\prime \prime}$, the approximate Nyquist frequency of the IRAS image. Figure 3 also shows evidence for a slowly varying background component, such as due to zodiacal or galactic emission, that appears as a nonzero intercept. As discussed in Section 3.1, the origin of the extended emission has been extensively investigated for IRAS data. This background, which appears as a constant offset in our small field, is not obviously related to the Taurus cloud so we do not attempt to model it here. Our temperature determination, which is based on the slope, does not require us to make any assumptions about the background level.

We find a dust temperature of $14.2 \pm 0.4 \mathrm{~K}$ for this part of the Taurus cloud (region shown in Figure 1), based on the slope of the fit (Figure 3 ) and assuming $\beta=2$ for the opacity power-law index. Color corrections were derived using the source emissivity from Equation (1) and are modest $(\sim 10 \%)$ for both MIPS and IRAS data at this temperature. The value given for $\beta=1.5$ indicates the uncertainty in the temperature if $\beta$ differs from the nominal value. An analysis using the completely independent COBE data (Lagache et al. 1998) shows excellent agreement; their Table 2 gives $T d=13.9 \pm 0.2 \mathrm{~K}$ at a position in common with our Taurus image.

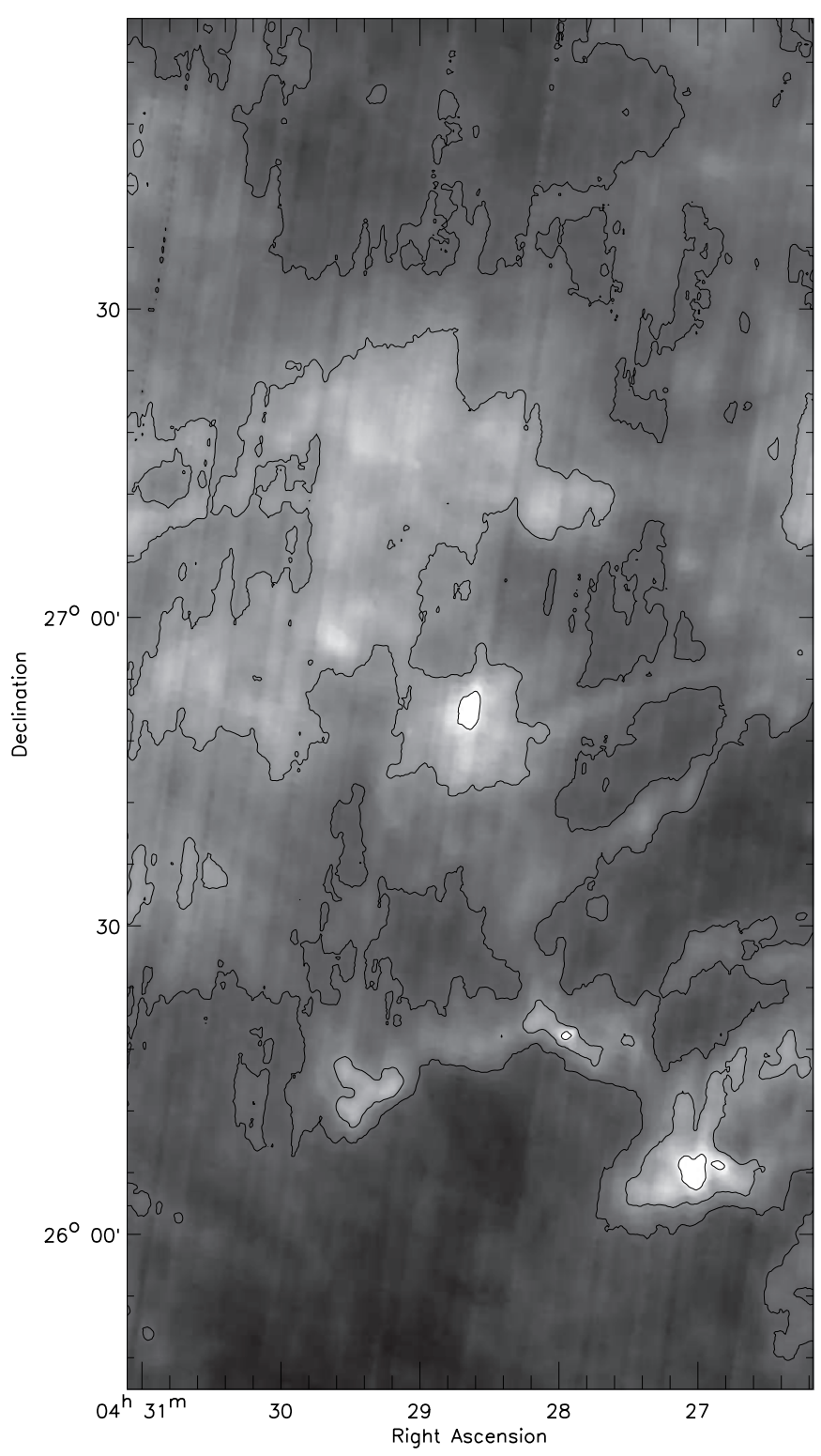

Figure 4. Optical depth map at $160 \mu \mathrm{m}$ using constant dust temperature (using Table 3). Solid lines show $0.0032,0.0044,0.008$ contour levels. If measured with respect to image minimum $(0.002$ at $04: 29: 12.725: 49: 13$ position) then the contours correspond to $\delta A_{V}=1,2,4$, respectively (using Table 4). Optical depths are less accurate at the highest contour level because the constant temperature assumption breaks down in these regions (see the text).

\subsection{Optical Depth and Opacity at $160 \mu \mathrm{m}$}

To the extent that the dust temperature is constant, then the structure seen at $160 \mu \mathrm{m}$ (Figure 1) represents variations in optical depth, i.e., variations in column density. Using Equation (1) and assuming a dust temperature of $14.2 \mathrm{~K}$, then a constant factor $6.18 \times 10^{-5}$ converts from intensity in $\mathrm{MJy} \mathrm{sr}^{-1}$ to $160 \mu \mathrm{m}$ optical depth (after background subtraction). Figure 4 shows the resulting $\tau_{160}$ optical depth map. Near the embedded YSOs, DG Tau, and IRAS 04248+2612, the dust temperature is higher than assumed so the optical depth will be overestimated within $3^{\prime}$ of the embedded sources. The optical depth can be underestimated in cold dense cores such as L1521F, a point that we return to.

In order to convert optical depth to column density (or mass) requires the opacity; however, the long-wavelength opacity 


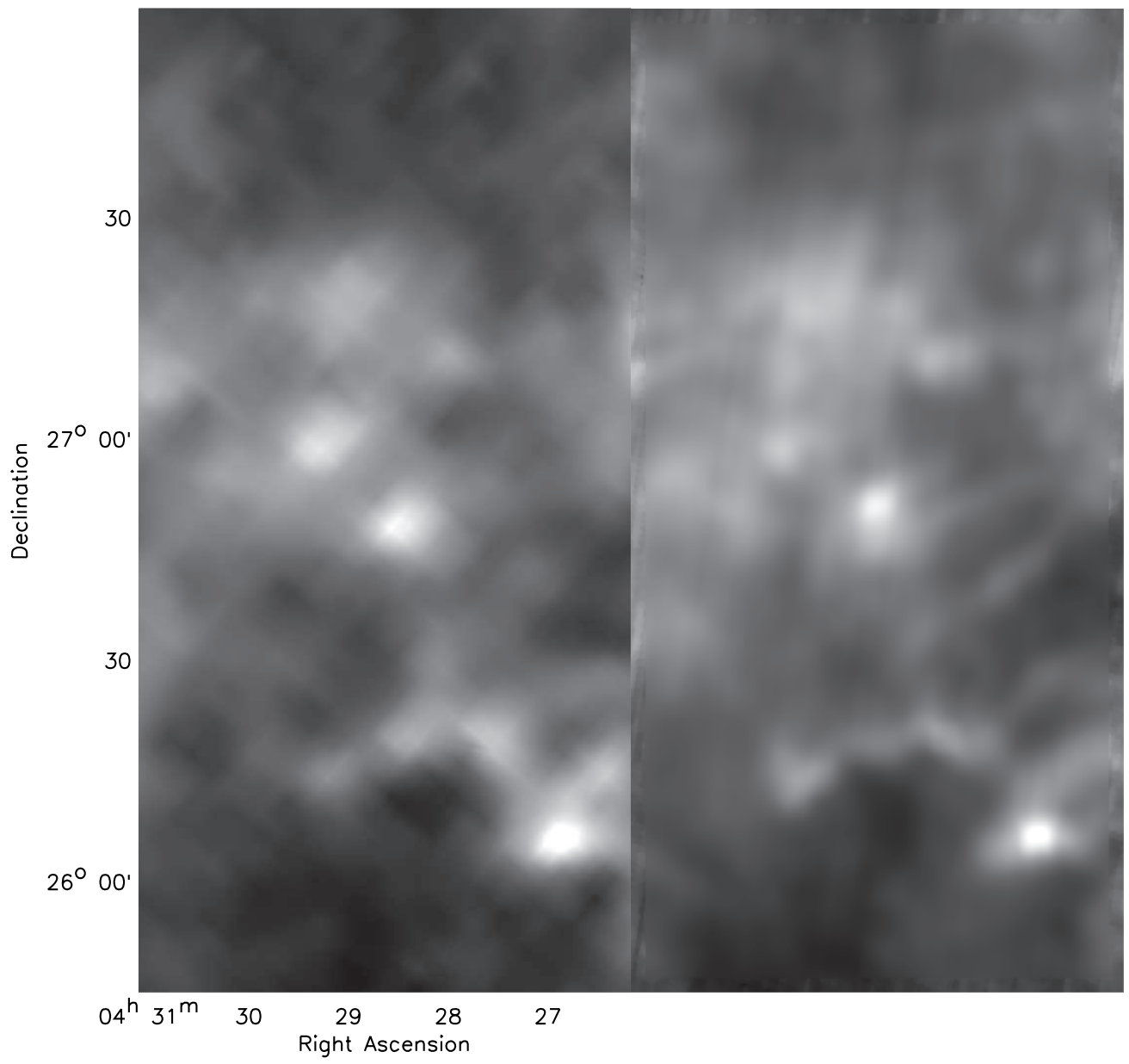

Figure 5. Comparison of extinction determined from 2MASS with that from $160 \mu \mathrm{m}$ data. The left panel shows $A_{V}$ from Froebrich et al. (2007); the right panel shows extinction $A_{160}$ (see Figure 4) but smoothed to have matching $4^{\prime}$ resolution.

is uncertain in molecular clouds, due to possible grain growth, ice mantle formation, and other factors (Ossenkopf \& Henning 1994; Stognienko et al. 1995; Evans et al. 2001). The situation is much better constrained for the diffuse ISM, where Lagache et al. (1999) found $\sigma=8.7 \pm 0.9 \times$ $10^{-26}(\lambda / 250 \mu \mathrm{m})^{-2} \mathrm{~cm}^{2} \mathrm{H}^{-1}$ using COBE data, which appears compatible with Weingartner \& Draine (2001) dust models. Scaling to the MIPS effective wavelength of $155.9 \mu \mathrm{m}$ then the diffuse ISM opacity is $2.3 \times 10^{-25} \mathrm{~cm}^{2} \mathrm{H}^{-1}$ from COBE data, which is close to the value of $2.0 \times 10^{-25} \mathrm{~cm}^{2} \mathrm{H}^{-1}$ for $R_{V}=3.1$ dust from Weingartner \& Draine (2001) and Draine (2003), and references therein. In alternate units (using $\mu=1.38$ ), the gas-dust opacity becomes $0.098 \mathrm{~cm}^{2} \mathrm{~g}^{-1}$ and $0.089 \mathrm{~cm}^{2} \mathrm{~g}^{-1}$, respectively. The opacity of dust-only is higher by a factor of the gas-to-dust ratio, the value of which is $M_{\text {gas }} / M_{\text {dust }}=124$ if using the models of Weingartner \& Draine (2001) and Draine (2003).

To test whether the diffuse ISM opacity applies in Taurus we compare the $160 \mu \mathrm{m}$ extinction with extinction $A_{V}$, which gives an independent measure of the column density. There is a strong correspondence (Figure 5) between images of $A_{V}$ at $4^{\prime}$ resolution from Two Micron All Sky Survey (2MASS; Froebrich et al. 2007) and MIPS $A_{160}$, smoothed to the same resolution. The trend seen in Figure 6 is linear, although it exhibits more scatter than is seen in Figure 3 for the dust temperature. The value of the slope (Table 4) is $A_{160} / A_{V}=0.0011$ which is 2.6 times higher than $A_{160} / A_{V}=0.00042$ for the diffuse ISM (Draine 2003;

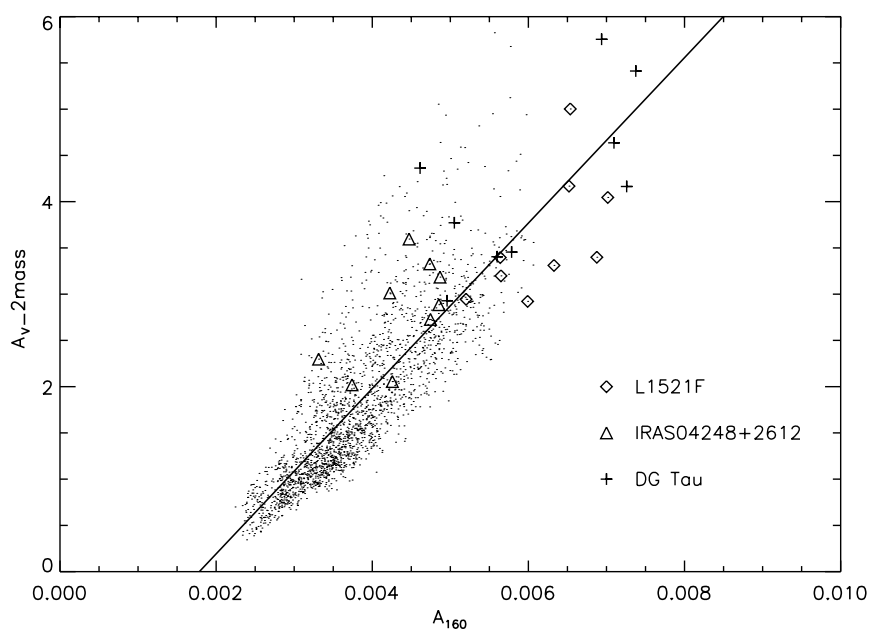

Figure 6. There is a linear correlation between $A_{V}$ and $A_{160}$ up to $A_{V} \sim 4$ for the spatial resolution matched data.

Weingartner \& Draine 2001). This comparison is based on dust with $R_{V}=3.1$, the kind found in the Taurus cloud (Kenyon et al. 1994); however, the predicted ratio hardly changes for $R_{V}=4.0$ or 5.5 dust models. Comparing the observed and theoretical ratios we conclude that the $160 \mu \mathrm{m}$ opacity in the Taurus molecular cloud is 2.6 higher than the diffuse ISM, namely $\sigma=5.2 \times 10^{-25} \mathrm{~cm}^{2} \mathrm{H}^{-1}$ and $\kappa=0.23 \mathrm{~cm}^{2} \mathrm{~g}^{-1}$ with 


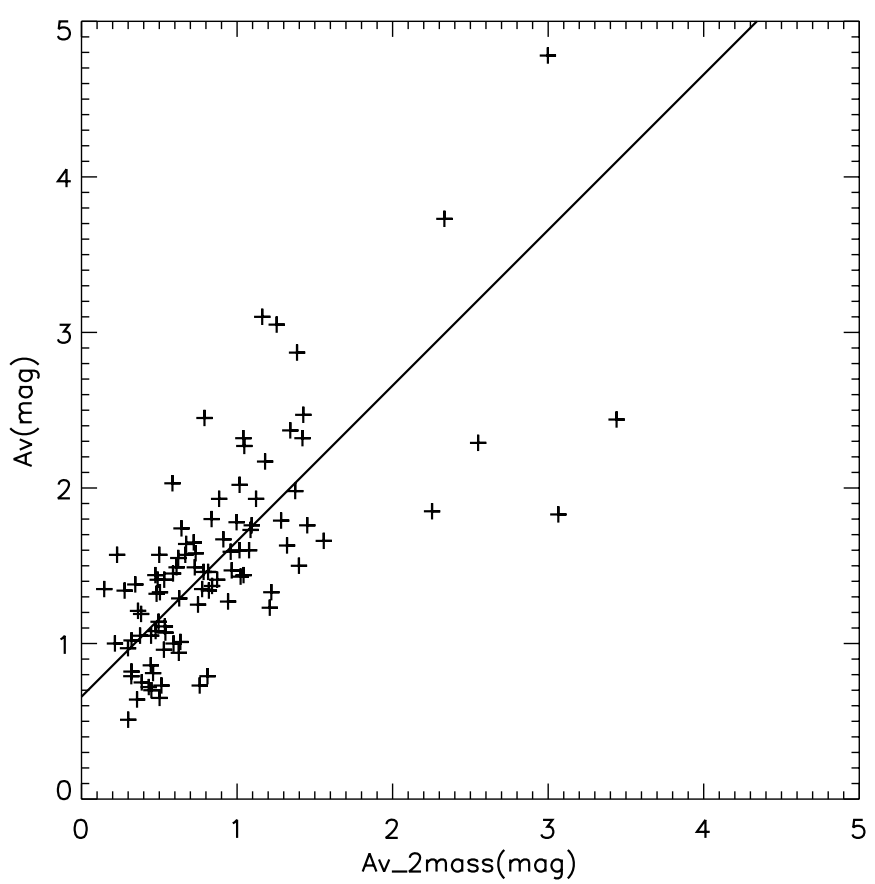

Figure 7. Comparison of two different $A_{V}$ methods shows good agreement with unit slope up to $A_{V} \sim 4$. However, the two methods have a systematic $0.66 \mathrm{mag}$ offset, and above $A_{V} \sim 2$ the scatter increases.

Table 4

Comparison of $160 \mu \mathrm{m}$ Opacity with $\mathrm{A}_{V}$

\begin{tabular}{cccccc}
\hline \hline $\begin{array}{l}I_{160} / A_{V}{ }^{\mathrm{a}} \\
\left(\mathrm{MJy} \mathrm{sr}^{-1} \mathrm{mag}^{-1}\right)\end{array}$ & $\begin{array}{c}A_{160_{0}}{ }^{\mathrm{a}} \\
(\mathrm{mag})\end{array}$ & $A_{160} / A_{V}{ }^{\mathrm{a}}$ & $A_{160} / A_{K}{ }^{\mathrm{a}}$ & $\begin{array}{c}\tau_{160} / N_{\mathrm{H}} \mathrm{b} \\
\left(\mathrm{cm}^{2} H^{-1}\right)\end{array}$ & $\begin{array}{c}\kappa_{160} \mathrm{~b} \\
\left(\mathrm{~cm}^{2} \mathrm{~g}^{-1}\right)\end{array}$ \\
\hline 18.1 & 0.0018 & 0.0011 & 0.010 & $5.2 \times 10^{-25}$ & 0.23 \\
\hline
\end{tabular}

Notes. Coefficients of linear fit to data shown in Figure 5.

${ }^{a}$ Estimated uncertainties are 10\% although formal errors are much smaller

${ }^{\mathrm{b}}$ Estimated 20\% uncertainties. Quantities at $160 \mu \mathrm{m}$ are derived based on $R_{V}=$ 3.1 dust model at wavelength $V$ and with model parameters $4.896 E-22=C_{\text {ext }}$ extinction cross section $\left(\mathrm{cm}^{2} H^{-1}\right), 1.870 E-26=M_{\text {dust }}$ per $\mathrm{H}$ nucleon $(\mathrm{g}$ $\mathrm{H}^{-1}$ ), $1.236 E+02=M_{\text {gas }} / M_{\text {dust }}, 0.6735=$ albedo (Draine 2003; Weingartner $\&$ Draine 2001)

estimated $1 \sigma$ error of $20 \%$. This indicates differences in Taurus cloud dust from diffuse ISM dust models, even in molecular cloud regions with modest $\left(A_{V}=0.4-4 \mathrm{mag}\right)$ extinction. Since the extinction maps of Froebrich et al. (2007) are in fact derived from near-IR data we express our result relative to $K$ band to find $A_{160} / A_{K}=0.010$, using $A_{V} / A_{K}=9.29$ from their assumed dust model. Flaherty et al. (2007) reported $A_{\lambda} / A_{K}$ values for shorter Spitzer wavelengths.

The results we present in Table 4 are fundamentally based on an empirical comparison of the $160 \mu \mathrm{m}$ intensity with $A_{V}$, hence we further assess the reliability of the $A_{V}$ determination. In their analysis of a nearby region in the Taurus cloud, Arce \& Goodman (1999) found significant differences between four different $A_{V}$ methods. In that work, they present $A_{V}$ based on spectral types for 95 stars; Figure 7 shows their $A_{V}$ values versus 2MASS derived $A_{V}$ from Froebrich et al. (2007). Comparison with a unit slope line shows good correspondence except for an offset, such that the 2MASS extinction is systematically 0.66 mag smaller than the $A_{V}$ based on spectral type. The offset seems plausible given the 2MASS derived value represents an average over $4^{\prime}$ resolution compared with the line-of-sight spectral value. We conclude that, apart from a nonzero offset,

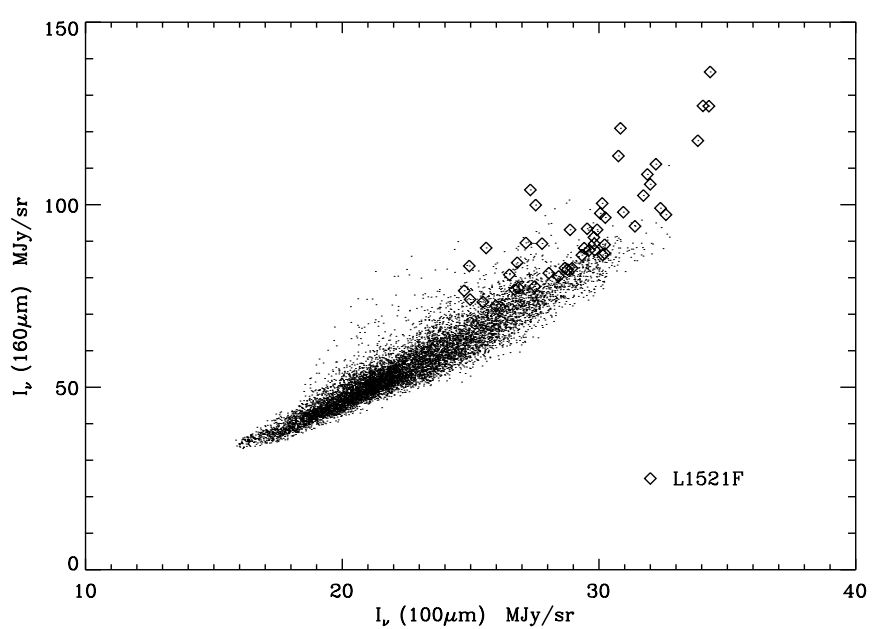

Figure 8. Using the unsmoothed $160 \mu \mathrm{m}$ data more clearly shows the $160 \mu \mathrm{m}$ excess from the cold dust in the L1521F core. Regions near the two embedded sources have been excluded.

the $A_{V}$ determination is approximately linear over the $A_{V}=$ $0.4-4$ mag range.

\subsection{Changes in Dust Temperature}

As shown in Figure 3, the dust temperature near two YSOs, IRAS $04248+2612$, and DG Tau, differs from the average $14.2 \mathrm{~K}$ value. The luminosities of 0.4 and $6.4 L_{\odot}$ for IRAS $04248+2612$ and DG Tau, respectively (White \& Hillenbrand 2004), further suggest that the dust "hot spots" are internally heated. We now consider the temperature near L1521F-IRS, a source that is much lower in luminosity. Figure 3 shows that data points in the L1521F core follow the average trend, and hence show no signature of dust heating. Since the heating effect might be small, in Figure 8 we also plot the unsmoothed $160 \mu \mathrm{m}$ versus the IRAS $100 \mu \mathrm{m}$ data from Figure 1, sampled at the $160 \mu \mathrm{m}$ resolution. The resulting increased structure at $160 \mu \mathrm{m}$ leads to increased vertical scatter, as is seen. However note that the points in the L1521F core all lie systematically above the other data points. This suggests a temperature effect leading to extra $160 \mu \mathrm{m}$ emission from colder than average dust. We conclude that there is no evidence for dust heating at 100 or $160 \mu \mathrm{m}$ by the embedded IR source in L1521F. We turn this statement into a flux density upper limit. Consider the peak emission value for $\mathrm{L} 1521 \mathrm{~F}$, namely the highest $160 \mu \mathrm{m}$ point in Figure 3 for L1521F. If this data point were moved horizontally to the right, increasing its $100 \mu \mathrm{m}$ intensity by about $3 \mathrm{MJy}$ $\mathrm{sr}^{-1}$ then we would plausibly conclude that there is evidence for dust heating. We use our empirical $100 \mu \mathrm{m}$ PSF to convert this intensity increment into an upper limit of $2 \mathrm{Jy}$ for the $100 \mu \mathrm{m}$ flux density from circumstellar heated dust around L1521FIRS. The situation is different at shorter wavelengths where the emission is dominated by warm circumstellar dust rather than the cold cloud. The emission at $60 \mu \mathrm{m}$ shows this behavior (see Figure 2), where a near pointlike source is detected, but not emission from the extended dense core.

Previous studies at longer wavelengths demonstrate the presence of cold dust in L1521F. Moreover, Kirk et al. (2005) determined a characteristic value of $10 \mathrm{~K}$ from fitting the observed spectral energy distribution for a number of dense cores. Temperatures of some well-determined Taurus cores range from 8 to $12 \mathrm{~K}$ (Kirk et al. 2007). The models of Evans et al. (2001) predict varying dust temperature such that the center of the prestellar core is the coldest region. The physical explanation is that the 


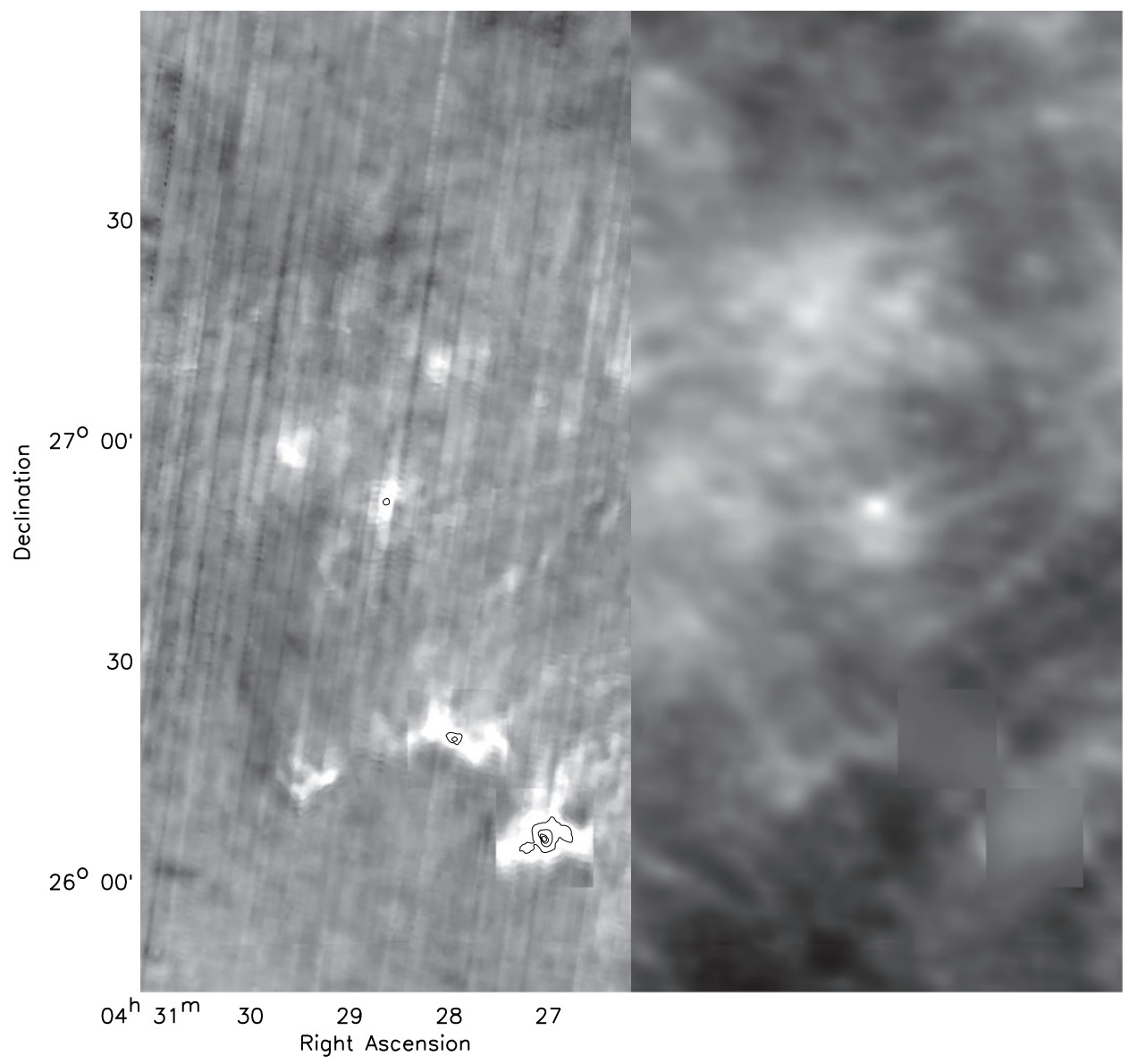

Figure 9. Optical depth grayscale maps at $160 \mu \mathrm{m}$ for core component (left) and cloud component (right). The $160 \mu \mathrm{m}$ excess map (left) traces regions that differ from the average behavior (right). The emission has been converted to optical depth for cores (left) assuming $T_{D}=10 \mathrm{~K}$ and for the cloud (right) using $T_{D}=14.2 \mathrm{~K}$. Adding the maps gives the total extinction. Optical depth contours start at 0.03 with 0.03 increment.

central region is partially shielded from the interstellar radiation field by the outer layers and thus can reach a lower temperature. Results from Kirk et al. (2007) give $9.3 \pm 0.3 \mathrm{~K}$ for the dust in the L1521F core. In addition, Codella et al. (1997) found a gas temperature of $T=9.1 \mathrm{~K}$ in $\mathrm{L} 1521 \mathrm{~F}$ using ammonia observations. The gas and dust temperatures are similar and plausibly suggest that the gas and dust may be well coupled at the $n \sim 10^{6} \mathrm{~cm}^{-3}$ density found in the L1521F cloud core.

\subsection{Optical Depth Maps and $160 \mu \mathrm{m}$ Cold Cores}

To accurately model the optical depth of dense cores requires knowing the dust temperature, which we know for L1521F but not for other cores since we lack longer wavelength data. Hence, we take a different approach to studying the cold core component. We a priori assume that two different temperature components contribute to the $160 \mu \mathrm{m}$ emission, namely $T=$ $10 \mathrm{~K}$ cores and $T=14.2 \mathrm{~K}$ clouds. We furthermore use the IRAS $100 \mu \mathrm{m}$ image to make our model of the cloud component. Thus, we obtain an excess image that is sensitive to the cold cores by subtracting a suitably scaled IRAS $100 \mu \mathrm{m}$ image from the MIPS $160 \mu \mathrm{m}$ image. Abergel et al. (1994) used a similar method in the Taurus cloud, but they use 60 and $100 \mu \mathrm{m} I R A S$ data, which is sensitive to somewhat warmer dust.

Figure 9 shows the resulting core and cloud images, each converted to optical depth using the assigned temperature. Including $10 \mathrm{~K}$ dust means the optical depth is significantly higher $(\times 14)$ in core regions than in Figure 4 . This implies that accurate optical depths for the cold cores depend on accurate dust temperatures. The dust temperature is known for $\mathrm{L} 1521 \mathrm{~F}$, but has not been measured for the other cores, which adds significantly to the uncertainty. Despite the caveats, the core excess image is quite useful. In Figure 9, the core image consists of features that appear at $160 \mu \mathrm{m}$ but not at $100 \mu \mathrm{m}$, while the cloud image looks like the $100 \mu \mathrm{m}$ image. Due to the ringing artifact, we treated the regions around the two bright YSOs differently. We cut out regions around the two point sources and filled them with median values determined over a larger region. The effective smoothing this procedure introduces means the optical depth near the two bright point sources has higher uncertainty. Moreover, as for Figure 4, optical depths very close to the point sources $\left(3^{\prime}\right)$ should be discounted, because those regions have warmer than average dust (see Figure 3).

One immediate use of the images is to identify dense cold cores that show up more clearly than in Figure 4. The core image shows six distinct features that we identify as dense cold cores. Three out of six cores contain embedded YSOs, the previously described L1521F-IRS, DG Tau, and IRAS 04248+2612. All six cores have counterparts in the optical extinction map catalog by Dobashi et al. (2005). The three cores containing YSOs were mapped by Onishi et al. (1998) and designated as $\mathrm{C}^{18} \mathrm{O}$ cores. Table 6 gives the $160 \mu \mathrm{m}$ cold core positions and crossidentifications.

\subsection{Properties of the L1521F Core}

To better understand our analysis we compare with previous studies of the L1521F core, starting with the extinction. The 
Table 5

IR Luminosity of L1527-IRS and L1521F-IRS

\begin{tabular}{lcccccccccc}
\hline \hline Source & $\lambda(\mu m)$ & 3.6 & 4.5 & 5.8 & 8.0 & 24 & 70 & 160 \\
\hline L1527-IRS & $f_{v}(m J y)$ & 1.2 & 6.2 & 11. & 8.2 & 580 & $29000^{\mathrm{b}}$ & $54000^{\mathrm{c}}$ & $\ldots$ \\
& $v L_{v}(\lambda)\left(L_{\odot}\right)$ & 0.0006 & 0.002 & 0.003 & 0.002 & 0.043 & 0.73 & 0.59 & 1.37 \\
& $v L_{v}(\lambda) / L_{\mathrm{TIR}}$ & $\ldots$ & $\ldots$ & $\ldots$ & 0.0001 & 0.03 & 0.53 & 0.43 & 1.0 & $\ldots .03$ \\
\multirow{2}{*}{ L1521F-IRS } & $f_{v}(\mathrm{mJy})$ & 0.20 & 0.35 & 0.48 & 0.98 & 24 & 460 & $\ldots$ & $\ldots$ \\
& $v L_{v}(\lambda)\left(L_{\odot}\right)$ & 0.0001 & 0.0001 & 0.0001 & 0.0002 & 0.002 & 0.011 & $0.010^{\mathrm{d}}$ & $0.024^{\mathrm{d}}$ \\
& $v L_{v}(\lambda) / L_{\mathrm{TIR}}$ & $\ldots$ & $\ldots$ & $\ldots$ & 0.009 & 0.07 & 0.49 & $0.43^{\mathrm{d}}$ & 1.0 \\
\hline
\end{tabular}

Notes. Fluxes from Table 2 or (D. L. Padgett et al. 2009, in preparation), unless noted. More L1527 fluxes in Tobin et al. (2008).

a Total IR luminosity, see the text.

b Source extended at $70 \mu \mathrm{m}$. Total flux density measured with standard $100^{\prime \prime}$ radius aperture, 120"-140" radius background, 1.13 aperture correction. Estimated $20 \%$ flux density error.

c Source extended at $160 \mu \mathrm{m}$ includes diffuse cloud. Central flux density measured with 64" radius aperture, 64"-96" radius background, background $=170 \mathrm{MJy} \mathrm{sr}^{-1}$ (i.e., extended cloud core), 1.38 aperture correction for $T<30 \mathrm{~K}$ sources. Estimated $20 \%$ flux density error.

${ }^{\mathrm{d}}$ Estimated value based on $L_{v} / L_{\mathrm{TIR}}=0.43$, adopted from $\mathrm{L} 1527$.

position of the IR source is spatially coincident with the peak of the extended emission at $160 \mu \mathrm{m}$; various studies show the core exhibits small-scale structure so therefore the peak extinction $A_{V}$ should depend on spatial resolution. The extinction based on 2MASS data (Froebrich et al. 2007) has the advantage that it covers the entire Taurus cloud. However, the achieved spatial resolution of $\sim 4^{\prime}$ is relatively low and in high extinction regions the method begins to break down leading to values that are systematically too low. This is borne out for L $1521 \mathrm{~F}$, where $A_{V}$ is the smallest we discuss: $A_{V}$ is 3.7 toward the IR source. The $1^{\prime}$ resolution of our $160 \mu \mathrm{m}$ image is higher, and for our single dust temperature fit (Figure 4) we find $A_{V}=8$ at the peak position. The $A_{V}$ drops to 6 if measured with respect to the image minimum, illustrating the uncertainty if a reasonable choice is made for a nonzero background level. We note that the assumed background level is not strongly dependent on image size, as the minimum in the L1521F image is close to the minimum found for the entire Taurus survey.

However, our analysis shows L $1521 \mathrm{~F}$ is one of the regions that contains colder than average dust. The relevant result is from Figure 9 and the extinction increases substantially for our two temperature model (Figure 9) to $A_{V}=43 \mathrm{mag}$, where the $10 \mathrm{~K}$ core contributes $38 \mathrm{mag}$, and the $14.2 \mathrm{~K}$ cloud contributes 5 mag to the total extinction. With background subtraction the $A_{V}$ drops to $41 \mathrm{mag}$, a small change. At yet higher (15") spatial resolution the trend of higher $A_{V}$ continues: Kirk et al. (2005) found a peak $A_{V}=130$ mag based on $850 \mu \mathrm{m}$ continuum data. In conclusion, we find that analysis of the $160 \mu \mathrm{m}$ emission provides a useful tool for determining $A_{V}$ in high extinction regions, although very accurate dust temperatures are needed to fully utilize the method.

Next we compute the mass of the L $1521 \mathrm{~F}$ core, and compare with previous studies. The mass $M$ is given by $M=\rho L A$, where $A$ is the projected area of the cloud core. Recalling that $\tau_{v}=\tau_{\lambda}, \kappa_{v}=\kappa_{\lambda}$ then comparison with Equation (2) shows that the mass is related to the optical depth such that

$$
\begin{aligned}
M= & A \tau_{\lambda} / \kappa_{\lambda}, \\
= & 0.61 M_{\odot}\left(0.23 \mathrm{~cm}^{2} \mathrm{~g}^{-1} / \kappa_{\lambda}\right)(D / 140 \mathrm{pc})^{2} \\
& \times\left(\Omega_{\text {pix }} / 8^{\prime \prime} \times 8^{\prime \prime}\right) \sum_{i} \tau_{160_{i}},
\end{aligned}
$$

where $\Omega_{\text {pix }}$ is the pixel solid angle in square arcseconds and the sum is over pixels within the dense core, and after background subtraction. One source of error in the mass is the opacity that
Table 6

Cross Identification of Spitzer $160 \mu \mathrm{m}$ Cold Cores

\begin{tabular}{lcccccc}
\hline \hline ID & $\begin{array}{c}\text { R.A. } \\
(\mathrm{deg})\end{array}$ & $\begin{array}{c}\text { Decl. } \\
(\mathrm{deg})\end{array}$ & $\begin{array}{c}l \\
(\mathrm{deg})\end{array}$ & $\begin{array}{c}b \\
(\mathrm{deg})\end{array}$ & $\begin{array}{c}A_{V} \text { Clump }^{\mathrm{a}} \\
\text { name }\end{array}$ & $\begin{array}{c}\mathrm{C}^{18} \mathrm{O} \mathrm{Core}^{\mathrm{b}} \\
\text { number }\end{array}$ \\
\hline 04:27:03+26:05:50 & 66.76 & 26.10 & 171.84 & -15.68 & $1211 \mathrm{P} 17$ & 21 \\
04:27:56+26:19:20 & 66.98 & 26.32 & 171.81 & -15.38 & $1211 \mathrm{P} 22$ & 22 \\
04:28:06+27:10:50 & 67.03 & 27.18 & 171.17 & -14.78 & $1211 \mathrm{P} 32$ & $\ldots$ \\
$04: 28: 39+26: 51: 40$ & 67.16 & 26.86 & 171.50 & -14.91 & $1211 \mathrm{P} 16$ & 20 \\
04:29:13+26:14:20 & 67.30 & 26.24 & 172.07 & -15.23 & $1211 \mathrm{P} 24$ & $\cdots$ \\
$04: 29: 35+26: 57: 30$ & 67.40 & 26.96 & 171.57 & -14.69 & $1211 \mathrm{P} 21$ & $\cdots$ \\
\hline
\end{tabular}

Notes. The $160 \mu \mathrm{m}$ peak position (J2000) using $15^{\prime \prime}$ pixels.

a Cloud name + clump name from 0.1 resolution catalog by Dobashi et al. (2005).

${ }^{\mathrm{b}}$ Core name from Table 1 in Onishi et al. (1998) and based on data with 0.05 resolution.

we previously estimated to have $20 \%$ uncertainty. The opacity models of Ossenkopf \& Henning (1994) treat dust coagulation and ice mantle formation in dense core environments. In their study of dense cores, Evans et al. (2001) considered dust opacities OH2 and OH5 from Ossenkopf \& Henning (1994), where they favor OH5 opacity (dust with thin ice mantles in $n=10^{6} \mathrm{~cm}^{-3}$ density gas). Both the $\mathrm{OH} 2$ and OH5 model opacities are consistent with our $160 \mu \mathrm{m}$ opacity; specifically for $M_{g} / M_{d}=124$ the model $\mathrm{OH} 2$ or OH5 leads to $0.25 \mathrm{~cm}^{2} \mathrm{~g}^{-1}$ or $0.33 \mathrm{~cm}^{2} \mathrm{~g}^{-1}$, respectively, both falling within $2 \sigma$ of our $0.23 \mathrm{~cm}^{2} \mathrm{~g}^{-1}$ value. How to compare our value with submillimeter opacities is less clear, but assuming $\beta=2$ and a gas-to-dust ratio of 100 instead of 124 gives $\kappa_{850}=$ $0.01 \mathrm{~cm}^{2} \mathrm{~g}^{-1}$ and $\kappa_{1200}=0.005 \mathrm{~cm}^{2} \mathrm{~g}^{-1}$, values that are about a factor of 2 lower than the OH5 opacities but are essentially the same as the opacities adopted by André et al. (1996), Crapsi et al. (2004) and Kirk et al. (2005). A second source of error is in finding the background level, that we estimate leads to $20 \%$ uncertainty in these extended objects. A third, and dominant, source of error is the temperature assumed for the cold cores. We adopted $10 \mathrm{~K}$ as a representative temperature for cold cores. However, Kirk et al. (2007) give $T=9.3 \pm 0.3 \mathrm{~K}$ for the L $1521 \mathrm{~F}$ core; using this lower temperature leads to a factor of 1.9 increase in the optical depth and hence the mass. Presumably the $9.3 \mathrm{~K}$ figure is more accurate, but we also quote masses using the $10 \mathrm{~K}$ dust temperature to illustrate the uncertainty that may apply to other cold cores found within our field.

The core mass determined by Crapsi et al. (2004) is $5.5 M_{\odot}$ from the $1.2 \mathrm{~mm}$ dust continuum; our measurement 
is 7.0, 3.7 $M_{\odot}$ for $9.3 \mathrm{~K}, 10 \mathrm{~K}$ dust temperature, respectively. The mass is calculated using $136^{\prime \prime}$ radius aperture, minus a background from a slightly larger radius to mimic the on-thefly mapping process. Similarly, Kirk et al. (2005) found a core mass of $2.6 M_{\odot}$ from the $850 \mu \mathrm{m}$ dust continuum within $75^{\prime \prime}$ radius aperture; within the same aperture we find $2.8,1.5 M_{\odot}$ for $9.3 \mathrm{~K}, 10 \mathrm{~K}$ dust temperature, respectively. Considering the difference in wavelength and techniques the agreement is good. However, in regions such as L $1521 \mathrm{~F}$ it is clear that an accurate dust temperature for the cold core is needed to determine an accurate mass.

We point out that the same value of $\kappa_{160}$ derived in modest $A_{V}=0.4-4$ regions seems to work equally well for the L 1521F dense core that contains both higher density and extinction. The opacity we determined for the Taurus cloud is higher than in the diffuse ISM, but we see no evidence for additional changes in the opacity within different molecular cloud environments.

\subsection{IR Luminosity of L1521F}

Standard methods of determining bolometric luminosity are problematic for low luminosity sources such as L1521F-IRS because the emission longward of $100 \mu \mathrm{m}$ is dominated by heating from the interstellar radiation field. To illustrate the difficulty, Boulanger \& Perault (1988) found an IR luminosity per solar mass of gas of $1.8 L_{\odot} / M_{\odot}$ for the diffuse ISM and $0.6 L_{\odot} / M_{\odot}$ in molecular clouds; the YSO luminosity thus represents an excess above this "background" luminosity. In the case of L1521F, prior to Spitzer, the source was successfully modeled purely as a externally heated prestellar core (Onishi et al. 1999; Crapsi et al. 2004), moreover whose emission longward of $100 \mu \mathrm{m}$ could be fit by a single temperature graybody (Kirk et al. 2005). The usual definition of bolometric luminosity means to integrate luminosity over all wavelengths. Here, we explicitly define $L_{\text {bol_int }}$ to mean just the portion of bolometric luminosity due to internal heating sources. To find $L_{\text {bol_int }}$ the approach taken by several authors has been to fit the source SED based on a physical model (Dunham et al. 2006; Bourke et al. 2006) and in this way, Bourke et al. (2006) found a best fit value of $0.05 \pm 0.02 L_{\odot}$ for L1521F-IRS. Although the method is promising, there is a concern that the applied correction to the observed IR luminosity is large and possibly model dependent. Dunham et al. (2008) further developed and tested this technique and suggested that $L_{\text {bol_int }}$ can be accurately estimated using the $70 \mu \mathrm{m}$ luminosity.

We adopt an alternative approach of reporting IR luminosities based solely on the Spitzer data, so that sources can be compared with each other in a robust way. We then introduce SED templates to estimate the internal bolometric luminosity. One immediate problem is that numerical integration of steeply rising SEDs is sensitive to how the boundary is treated. To avoid this difficulty we adopt several extragalactic conventions (Sanders \& Mirabel 1996) in part because galaxy SEDs share many similarities with embedded YSO SEDs. Specifically, $L_{\nu}(\lambda)=4 \pi D^{2} f_{v}$ is defined to be the luminosity per unit frequency. The total IR luminosity is then a weighted sum of $\gamma \nu L_{v}$ over specified bands, where $\gamma$ is a coefficient of order unity. Several definitions of the total IR luminosity, $L_{\mathrm{TIR}}$, have been proposed (see Huynh et al. 2007, and references therein). We adopt $L_{\mathrm{TIR}}=\gamma_{1} v L_{v}(8.0 \mu \mathrm{m})+\gamma_{2} v L_{v}(24 \mu \mathrm{m})+$ $\gamma_{3} v L_{v}(70 \mu \mathrm{m})+\gamma_{4} v L_{v}(160 \mu \mathrm{m})$ with $\gamma=[1,1,1,1]$ for simplicity, which is very similar to the $\gamma=[0.95,1.15,1,1]$ proposed by Draine \& Li (2007).

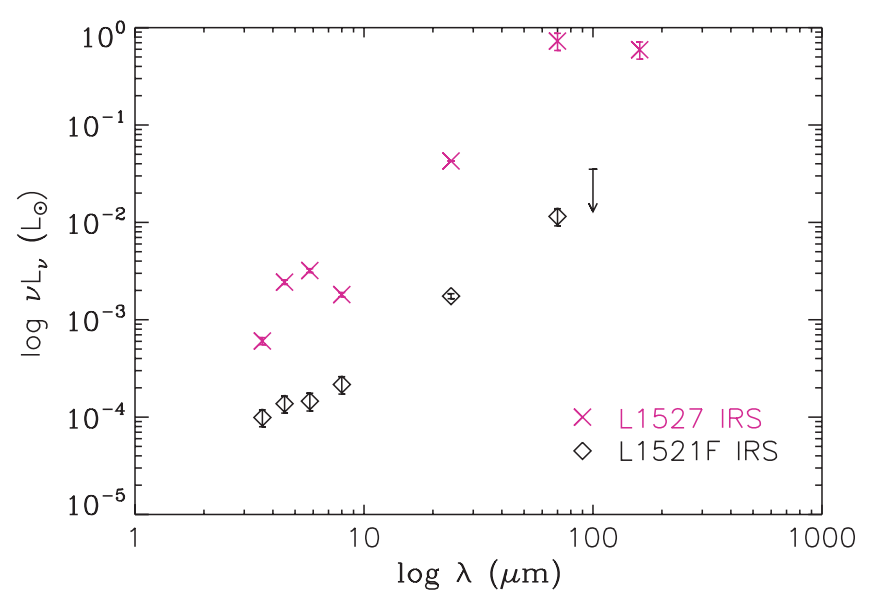

Figure 10. Comparison of class 0 and VeLLO spectral energy distributions. The top SED shows the central point source of L1527-IRS from $3.6 \mu \mathrm{m}$ to $160 \mu \mathrm{m}$. The dip at $8.0 \mu \mathrm{m}$ is associated with $10 \mu \mathrm{m}$ silicate absorption that is due to the edge-on source inclination (Furlan et al. 2008; Tobin et al. 2008). The overall SED of L1521F-IRS is lower indicating a lower luminosity. Both sources have steeply rising SEDs showing they are deeply embedded.

One remaining task is to estimate the flux density for $\mathrm{L} 1521 \mathrm{~F}$ at $160 \mu \mathrm{m}$ since we detected only the cloud but not the embedded source at this wavelength. We do this by generating an SED template for class 0 sources, using for our template the well-known class 0 source L1527-IRS (IRAS 04368+2557) in Taurus. Because L1527-IRS is an extended source at $160 \mu \mathrm{m}$ we briefly discuss its flux density measurement. The flux density is 54 Jy using 64" radius aperture with background determined from a just-larger annulus. The choice of aperture and background is important, and to some extent arbitrary, which seems unavoidable. For L1527-IRS the measured half-width at half-maximum (HWHM) size at $160 \mu \mathrm{m}$ is $50^{\prime \prime}$, meaning the $64^{\prime \prime}$ aperture is slightly larger. To compare with KAO measurements of Ladd et al. (1991) we use the background at 300" ; our flux density then increases to 76 Jy (estimated $20 \%$ uncertainty), which compares well with the total flux of $94 \pm 33$ Jy found by Ladd et al. (1991). In our SED, we use the 54 Jy flux density as the value that better represents the circumstellar emission. Table 5 contains measured flux density values for L1527-IRS, while Figure 10 shows the SED.

The total IR luminosity $L_{\mathrm{TIR}}=1.4 L_{\odot}$ is dominated by the 70 and $160 \mu \mathrm{m}$ fluxes, and moreover compares favorably with the IRAS result $L_{I R A S}=1.6 L_{\odot}$ (Kenyon et al. 1990). Note that the different beam sizes, with IRAS being the larger $\left(5^{\prime}\right)$, likely account for any differences. The source has been recently modeled by several groups. Furlan et al. (2008) reported $L_{\text {bol_int }}=1.8 L_{\odot}$ for the star plus disk luminosity from a model fit to the SED. Tobin et al. (2008) presented extensive modeling of the SED and IRAC images of this edge-on source. They determine $L_{\text {bol_int }}=2.0 L_{\odot}$ from integrating an aperturedefined model SED, and furthermore note that not all photons appear in the SED so the internal luminosity is in fact higher, leading to $2.6 L_{\odot}$ for the total value. In terms of the SED fit, the model undershoots the long-wavelength part of the SED, which suggests that external heating by the ISRF is important, even for this relatively luminous YSO. To conclude, the Spitzer luminosity is $L_{\mathrm{TIR}}=1.4 L_{\odot}$ and based on the models, the internal bolometric luminosity ranges from $\sim 1.9 L_{\odot}$ to $2.6 L_{\odot}$ which implies a multiplicative bolometric correction of 1.4-1.9, respectively.

Returning to L1521F-IRS we find $L_{\mathrm{TIR}}=0.024 L_{\odot}$ (Table 5) where we have estimated $\nu L_{\nu}(160 \mu \mathrm{m})$ using the 

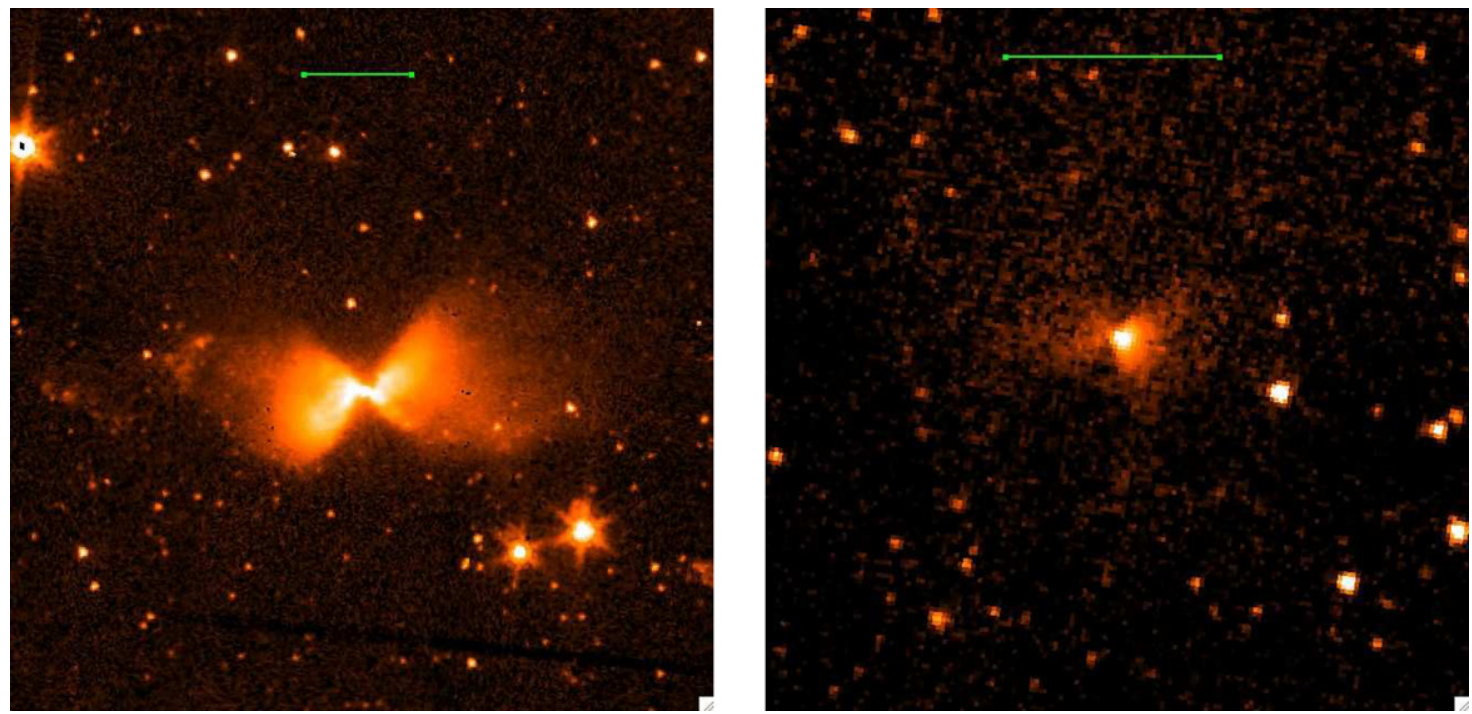

Figure 11. Comparison of class 0 and VeLLO images at IRAC $4.5 \mu$ m wavelength shows scattered light nebulosity toward both. Scale in green is $1^{\prime}=8400$ AU. North is up, east to the left. Left: image of L1527-IRS edge-on system shows a disk oriented N-S and outflow cavity E-W in scattered light. Right: L1521F-IRS lies at apex of conical nebulosity, suggesting an outflow seen at the moderate inclination. The smaller extent of the VeLLO nebulosity is consistent with its lower luminosity.

SED template. The estimated bolometric luminosity is then $L_{\text {bol_int }}=0.034-0.046 L_{\odot}$, reflecting the uncertainty in the bolometric correction. This value is consistent with the luminosity of $0.05 \pm 0.02 L_{\odot}$ found by the Bourke et al. (2006) model and $L_{i n t}=0.03 L_{\odot}$ from Dunham et al. (2008). Our result confirms the extremely low luminosity of the source.

\subsection{Comparison of L1521F-IRS with the Class 0 Source L1527}

We continue our comparison of L1521F-IRS with L1527 (IRAS $04368+2557$ ), the one and only previously known class 0 that falls within the boundary of the $44 \mathrm{deg}^{2}$ Taurus Spitzer Legacy Survey. Neither source was detected by 2MASS at $2.2 \mu \mathrm{m}$, consistent with their deeply embedded nature. However, the lower extinction at IRAC wavelengths $(3.6-8 \mu \mathrm{m})$ and high sensitivity mean that Spitzer is sensitive to the outflow, showing reflection nebulosity with bipolar morphology toward both sources (Bourke et al. 2006; Tobin et al. 2008).

Figure 11 shows the two objects side by side and illustrates the difference in both spatial extent and source inclination. The symmetric morphology of L1527-IRS implies an edge-on $i \sim 80^{\circ}-90^{\circ}$ inclination, that is confirmed by an $8 \mu \mathrm{m}$ dip in the SED (Figure 10) that is due to deep silicate absorption (Furlan et al. 2008; Tobin et al. 2008). By contrast, the morphology of L1521F-IRS in both our data and that of Bourke et al. (2006) finds the source near the apex of a conical, rather than a symmetric bi-conical structure at 3.6, 4.5, and $5.8 \mu \mathrm{m}$; the fact that one outflow lobe is much brighter than the other in IRAC images implies that the source has a moderate inclination (Whitney et al. 2003; Terebey et al. 2006). The radiative transfer models of Whitney et al. (2003) and references therein show the importance of inclination on the SED and images. Based on our simulated images of a protostar with an opaque envelope (Terebey et al. 2006) we estimate the inclination range is $i \sim 50^{\circ}-70^{\circ}$ for L1521F-IRS. The smaller spatial extent as well as the moderate inclination of L1521F-IRS further show that its luminosity is intrinsically low rather than low due to an inclination effect.

The 1' scale bar in Figure 11 shows the approximate diameter of the infalling region, where the radius is given by $r=a t$ for a sound speed $a=0.2 \mathrm{kms}^{-1}$ (appropriate for Taurus) and time $t=10^{5} \mathrm{yr}$ (Terebey et al. 1984; Shu et al. 1987). The age of L1527 is not well known but recent results from the c2d Spitzer Legacy Survey give $1.3 \times 10^{5} \mathrm{yr}$ for the class 0 lifetime (Evans et al. 2009). The scale bar thus shows the approximate size of the collapsing cloud for class 0 objects. If the VeLLO source were the same age, then in the context of low-mass star-formation theory the mass of the collapsing region would be about $2 M_{\odot}$ for the same size region (see Section 3.7).

\section{DISCUSSION}

\subsection{Evolutionary Status of L1521F-IRS}

The interest of L1521F-IRS and other VeLLO sources is where they fit into the evolutionary scheme of low-mass star formation. Theory predicts an extended accretion phase, the embedded phase, during which a protostar grows in mass, and hence, luminosity, and displays the morphology of infalling cloud core (aka infall envelope), circumstellar disk, and outflow (Terebey et al. 1984; Shu et al. 1987). The embedded phase is represented by class 0 and I sources, where class 0 s appear younger, based in part on being more deeply embedded but nonetheless having a central component capable of driving an outflow (André et al. 1993).

Although much lower in luminosity, VeLLOs share many similarities to known class 0 sources, including a steeply rising SED and evidence for outflows. For the purpose of comparison we consider VeLLO sources separately from class 0 sources, as their evolutionary status is still being defined. For the VeLLO L1521F and class $0 \mathrm{~L} 1527$, the cloud core masses are similar but the total IR luminosity of L1521F-IRS $\left(0.024 L_{\odot}\right)$ is roughly 50 times smaller. The low luminosity of L1521F-IRS demonstrates that it has a low central mass, regardless of whether the luminosity is dominated by $L_{*}$, the central object luminosity, or $L_{\text {acc }}=f_{x} G M_{*} \dot{M}_{*} / R_{*}$, the accretion luminosity. Because the accretion luminosity is proportional to stellar mass, one interpretation for the low luminosity of L1521F-IRS is that it may be younger than L1527-IRS provided the mass infall rates are similar. Given the likely importance of initial conditions at this early phase it seems unlikely that the assumption of constant mass infall rate applies, so it would be an overinterpretation of theory to say that L1521F-IRS is 50 times younger. Stamatellos 
et al. (2005) predicted luminosities above $1 L_{\odot}$ and would thus suggest VeLLO sources are late class I phase. However, the infall rates in Stamatellos et al. (2005) are high compared with typical values for Taurus and the model duration is relatively short $1.6 \times 10^{4} \mathrm{yr}$ to form a $0.5 M_{\odot}$ protostar. Using infall rates appropriate for Taurus, we argue that the morphology and luminosity suggest that L1521F-IRS could be younger or comparable in age to the class $0 \mathrm{~L} 1527$, while the similar mass reservoir of the cloud cores suggests that over time L1521F-IRS may eventually accrete enough mass to become a full-fledged star. The cloud core has no well-defined edge, but contains at least $5 M_{\odot}$, thus the source tests the idea that the final stellar mass is determined by the available mass reservoir and will approach a relatively constant fraction of the core mass (Motte et al. 1998; Onishi et al. 1998; Testi \& Sargent 1998; Johnstone et al. 2001; Alves et al. 2007; Enoch et al. 2008).

At this point the statistics in Taurus suggest similar numbers of VeLLO and previously known class 0 sources in Taurus. To distinguish the two types we choose a luminosity boundary of $L_{\mathrm{TIR}}=0.5 L_{\odot}$, which corresponds to roughly $L_{\text {bol_int }}=$ $1.0 L_{\odot}$. To motivate this choice we calculate that $M_{*}=0.1 M_{\odot}$ leads to an accretion luminosity $L_{\text {acc }}=1.0 L_{\odot}$, assuming a standard infall rate of $1.6 \times 10^{-6} M_{\odot} \mathrm{yr}^{-1}$, of which fraction $f_{m}=2 / 3$ falls onto a protostar of radius $1 R_{\odot}$ (Figure 7 of Stahler (1988)) and is converted to accretion luminosity with $f_{x}=0.62$ efficiency (Shu et al. 1996). Further discussion of efficiencies appears in Terebey et al. (2006). For sources whose luminosity is dominated by the accretion luminosity, the implied mass $M_{*}=0.1 M_{\odot}$ is close to the stellar-substellar boundary, so this choice of luminosity boundary distinguishes between objects that are stellar rather than substellar.

In addition to L1521F and L1527, which lie within the Legacy Survey boundaries, there are two objects in the southern part of Taurus, namely the VeLLO IRAM 04191+1522 and the suggested transitional class 0/I source L1551NE (not to be confused with L1551 IRS5) (André et al. 1999; Dunham et al. 2006; Moriarty-Schieven et al. 1995). If the star-formation rate has been constant in Taurus then equal numbers imply equal time duration for the two types. Admittedly the numbers are so small that conclusions are premature, except to say that there is no evidence in Taurus that VeLLOs represent a short-lived (10× shorter) phase compared with current timescale estimates for class 0 objects. The survey results of Dunham et al. (2008) present 15 new VeLLO candidates, so there is a promise of much better statistics in the near future.

Existing theoretical simulations of the first protostellar core phase appear to be incompatible with the observations of L1521F-IRS. This is because the first protostellar core is relatively cold and produces little or no emission shortward of $70 \mu \mathrm{m}$ (Boss \& Yorke 1995; Bate 1998; Stamatellos et al. 2005; Saigo et al. 2008), whereas L1521F-IRS is well detected at all IRAC wavelengths. The next phase, the formation of the second (hydrostatic) core, produces emission at the requisite IRAC wavelengths. However, the existing simulations use high infall rates, and hence produce accretion luminosity $L>1 L_{\odot}$, which is many times higher than the luminosity of L1521FIRS. A lower infall rate is plausible, hence the source L1521FIRS might represent the beginning of the second protostellar core phase. There is also the possibility that infall rates are variable. If the current infall rate is very low then the accretion luminosity can drop below $L_{*}$ for the central object. Theoretical evolutionary tracks are relevant for this case although still highly uncertain at young ages. The tracks presented in Baraffe et al. (2002) suggest that $0.1 M_{\odot}$ is the maximum possible mass for
$L<0.1 L_{\odot}$ if the age is less than 1 Myr. From this we conclude that the current mass of L1521F-IRS is likely to be substellar.

Returning to Figure 11, we resume our comparison of the VeLLO and class 0 sources. It is clear that L1527 and L1521FIRS are different now (extent of nebulosity, luminosity, etc.). However both sources could, in fact, have the same (substellar) mass and their differences plausibly be explained by very different accretion rates onto the central protostar (e.g., Terebey et al. 2006). If the accretion of L1527 were "turned off," or the accretion onto L1521F IRS "ramped up" then they could have the same SED, luminosity, $\mathrm{CO}$ outflow, and spatial extent. If the accretion rate were, for example, time variable then L1527 could represent a high-phase, and L1521F-IRS a low-phase during class 0 evolution.

The data presented here do not offer a conclusive statement about the final mass of the protostar in L1521F-IRS, whether it will remain a substellar object or continue to grow in mass, as the L1527 class 0 source appears likely to do. However, L1521FIRS is embedded near the center of a collapsing cloud core (Onishi et al. 1999; Lee et al. 2004), and it is likely to continue to accrete material (possibly at a variable rate) from an ample mass reservoir until infall is terminated by one of possibly many physical processes (Terebey et al. 1984; Shu et al. 1987; Myers 2008; Evans et al. 2009). Given its location inside the cloud core, we suggest that L1521F-IRS will not remain substellar unless some condition, such as high angular momentum or ejection, successfully terminates accretion soon.

\section{CONCLUSIONS}

Using data from the Taurus Spitzer Legacy Survey, we analyze the $160 \mu \mathrm{m}$ far-IR emission near L1521F to investigate the cloud environment and look for heating by the central object. The L1521F prestellar core contains a very low luminosity embedded IR source previously detected by the SST. The source's low luminosity suggests the object is substellar, and its similarity to class 0 sources suggests it is extremely young.

We investigate the influence of L1521F-IRS on its natal cloud by studying the Spitzer MIPS $160 \mu \mathrm{m}$ and IRAS $100 \mu \mathrm{m}$ data in a $1^{\circ} \times 2^{\circ}$ region to look for evidence of dust heating at these wavelengths. In Figures 1, 3, and 5, we present the basic data and show that (1) the emission is dominated by a uniform dust temperature of $14.2 \mathrm{~K}$ except near two bright YSOs in the field and (2) there is no evidence for any dust heating associated with L1521F-IRS, suggesting that its impact on the dust continuum at $\lambda>100 \mu \mathrm{m}$ is minimal. Note however that at $70 \mu \mathrm{m}$ the dust emission is dominated by the pointlike embedded source.

The cloud dust temperature is $14.2 \pm 0.4 \mathrm{~K}$, based on the observed linear correlation between the MIPS $160 \mu \mathrm{m}$ and IRAS $100 \mu \mathrm{m}$ data. There is also a linear correlation with extinction up to $A_{V} \sim 4$, from which we derive $A_{160} / A_{K}=0.010 \pm 0.001$ for the extinction ratio and $\kappa_{160}=0.23 \pm 0.046 \mathrm{~cm}^{2} \mathrm{~g}^{-1}$ for the specific opacity of the gas-to-dust mixture. The value of the opacity at $160 \mu \mathrm{m}$ is consistent with dust models that incorporate dust coagulation and/or ice mantle formation in dense core environments (Ossenkopf \& Henning 1994). The $160 \mu \mathrm{m}$ opacity is 2.6 times higher than that measured for the diffuse ISM, but is consistent with predictions for dense cores, which suggests that there are significant differences between dust in the Taurus cloud and the diffuse ISM even at modest extinction values.

The MIPS $160 \mu \mathrm{m}$ data show evidence for a cold core component that is not visible in the IRAS $100 \mu \mathrm{m}$ data. The analysis produces an excess map at $160 \mu \mathrm{m}$ that is sensitive 
to colder regions, namely cold dense cores. In the subregion studied we find six cold cores, all of which have counterparts in extinction maps or $\mathrm{C}^{18} \mathrm{O}$ maps. By assuming $10 \mathrm{~K}$ dust temperature for the cold cores, we compute the optical depth at $160 \mu \mathrm{m}$ for both the cloud (14.2 K) and core (10 K) components. Three of the six cold cores contain embedded YSOs and thus indicate that the cold cores are regions of current low-mass star formation.

To measure source luminosity in an alternate way to model fitting, we introduce the Spitzer total IR luminosity $L_{\mathrm{TIR}}$ and make use of SED templates. For L1521F-IRS, $L_{\mathrm{TIR}}=0.024 L_{\odot}$ and $L_{\text {bol_int }}=0.034-0.046 L_{\odot}$, depending on the bolometric correction. This result confirms the low source luminosity of $L_{\text {bol_int }}=0.05 L_{\odot}$ determined by Bourke et al. (2006) and $L_{\text {int }}=0.03 L_{\odot}$ found by Dunham et al. (2008). In addition, we estimate the source inclination is $50^{\circ}-70^{\circ}$ based on the morphology of the extended emission in the IRAC images.

To date the Taurus Spitzer Survey contains the known class 0 source L1527-IRS, as well as the VeLLO source L1521F-IRS. The source L1521F-IRS appears to be a substellar object that is at a similar or possibly younger evolutionary state than the class 0 in L1527. The mass reservoir of roughly $2-5 M_{\odot}$ in the core suggests that $\mathrm{L} 1521 \mathrm{~F}$ is capable of eventually becoming a solarmass type star. Comparison of L1521F-IRS with theoretical models for the very early phases of star-formation rule out the first core collapse phase. However, it may be consistent with the early second collapse phase, defined as accretion onto a very low-mass hydrostatic object. Alternatively, if the infall rate is variable, and currently low, then the implied mass could be higher. However the low source luminosity is difficult to explain unless the source is substellar.

This work is based in part on observations carried out by the Spitzer Space Telescope, which is operated by the Jet Propulsion Laboratory, California Institute of Technology, under NASA contract 1407. S.T. warmly thanks the Spitzer Science Center, UCLA Department of Astronomy, Caltech Department of Astronomy, and Harvard-Smithsonian Center for Astrophysics for their hospitality. M.A. acknowledges support from a Swiss National Science Foundation Professorship (PP002-110504).

Facilities: SSO, IPAC (IRAS)

\section{REFERENCES}

Abergel, A., Boulanger, F., Mizuno, A., \& Fukui, Y. 1994, ApJ, 423, L59

Alves, J., Lombardi, M., \& Lada, C. J. 2007, A\&A, 462, L17

André, P., Motte, F., \& Bacmann, A. 1999, ApJ, 513, L57

André, P., Ward-Thompson, D., \& Barsony, M. 1993, ApJ, 406, 122

André, P., Ward-Thompson, D., \& Motte, F. 1996, A\&A, 314, 625

Arce, H. G., \& Goodman, A. A. 1999, ApJ, 517, 264

Baraffe, I., Chabrier, G., Allard, F., \& Hauschildt, P. H. 2002, A\&A, 382, 563

Bate, M. R. 1998, ApJ, 508, L95

Boss, A. P., \& Yorke, H. W. 1995, ApJ, 439, L55

Boulanger, F., Beichman, C., Desert, F. X., Helou, G., Perault, M., \& Ryter, C. 1988, ApJ, 332, 328

Boulanger, F., \& Perault, M. 1988, ApJ, 330, 964

Bourke, T., et al. 2006, ApJ, 649, L37

Cao, Y., Terebey, S., Prince, T. A., \& Beichman, C. A. 1997, ApJS, 111, 387

Codella, C., Welser, R., Henkel, C., Benson, P. J., \& Myers, P. C. 1997, A\&A, 324,203

Crapsi, A., Caselli, P., Walmsley, C. M., Myers, P. C., Tafalla, M., Lee, C. W., \& Bourke, T. L. 2005, ApJ, 619, 379

Crapsi, A., Caselli, P., Walmsley, C. M., Tafalla, M., Lee, C. W., Bourke, T. L., \& Myers, P. C. 2004, A\&A, 420, 957

di Francesco, J., Evans, N. J., II, Caselli, P., Myers, P. C., Shirley, Y., Aikawa, Y., \& Tafalla, M. 2007, in Proc. Protostars \& Planets V, ed. B. Reipurth, D. Jewitt, \& K. Keil (Tucson, AZ: Univ. Arizona Press), 17
Dobashi, K., Uehara, H., Kandori, R., Sakurai, T., Kaiden, M., Umemoto, T., \& Sato, F. 2005, PASJ, 57, S1

Draine, B. T. 2003, ARA\&A, 41, 241

Draine, B. T., \& Li, A. 2007, ApJ, 657, 810

Dunham, M. M., Crapsi, A., Evans, II, N. J., Bourke, T. L., Huard, T. L., Myers, P. C., \& Kauffmann, J. 2008, ApJS, 179, 249

Dunham, M. M., et al. 2006, ApJ, 651, 945

Enoch, M. L., Evans, II, N. J., Sargent, A. I., Glenn, J., Rosolowsky, E., \& Myers, P. 2008, http://arxiv.org/abs/0805.1075

Evans, N. J., II 1999, ARA\&A, 37, 311

Evans, N. J., II, Rawlings, J. M. C., Shirley, Y. L., \& Mundy, L. G. 2001, ApJ, 557, 193

Evans, N. J., et al. 2009, ApJS, 181, 321

Fazio, G. G., et al. 2004, ApJS, 154, 10

Flaherty, K. M., Pipher, J. L., Megeath, S. T., Windston, E. M., Gutermuth, R. A., Muzerolle, J., Allen, L. E., \& Fazio, G. G. 2007, ApJ, 663, 1069

Froebrich, D., Murphy, G. C., Smith, M. D., Walsh, J., \& del Burgo, C. 2007, MNRAS, 378, 1447

Furlan, E., et al. 2008, ApJS, 176, 184

Huard, T. L., et al. 2006, ApJ, 640, 391

Huynh, M. T., Frayer, D. T., Mobasher, B., Dickenson, M., Chary, R.-R., \& Morrison, G. 2007, ApJ, 667, L9

Johnstone, D., Fich, M., Mitchell, G. F., \& Moriarty-Schieven, G. 2001, ApJ, 559,307

Kenyon, S. J., Dobrzycka, D., \& Hartman, L. 1994, AJ, 108, 1872

Kenyon, S. J., Hartmann, L. W., Strom, K. M., \& Strom, S. S. 1990, AJ, 99, 869

Kirk, J. M., Ward-Thompson, D., \& André, P. 2005, MNRAS, 360, 1506

Kirk, J. M., Ward-Thompson, D., \& André, P. 2007, MNRAS, 375, 843

Ladd, E. E., Adams, F. C., Casey, S., Davidson, J. A., Fuller, G. A., Harper, D A., Myers, P. C., \& Padman, R. 1991, ApJ, 382, 555

Lagache, G., Abergel, A., Boulanger, F., Desert, F. X., \& Puget, J.-L. 1999, A\&A, 344,322

Lagache, G., Abergel, A., Boulanger, F., \& Puget, J.-L. 1998, A\&A, 333, 709

Lee, C. W., \& c2d Team, 2005, in Proc. Protostars \& Planets V, 8085

Lee, C. W., Myers, P. C., \& Plume, R. 2004, ApJS, 153, 523

Li, A., \& Draine, B. T. 2001, ApJ, 554, 778

Milville-Deschenes, M.-A., \& Lagache, G 2005, ApJS, 157, 302

Moriarty-Schieven, G. H., Butner, H. M., \& Wannier, P. G. 1995, ApJ, 445, L55

Motte, F., Andre, P., \& Neri, R. 1998, A\&A, 336, 150

Myers, P. C. 2008, ApJ, 687, 340

Onishi, T., Mizuno, A., \& Fukui, Y. 1999, PASJ, 51, 257

Onishi, T., Mizuno, A., Kawamura, A., Ogawa, H., \& Fukui, Y. 1998, ApJ, 502, 296

Ossenkopf, V., \& Henning, T. 1994, A\&A, 291, 943

Rieke, G. H., et al. 2004, ApJS, 154, 25

Saigo, K., Tomisaka, K., \& Matsumoto, T. 2008, ApJ, 674, 997

Sanders, D. B., \& Mirabel, I. F. 1996, ARA\&A, 34, 749

Shinnaga, H., Ohashi, N., Lee, S.-W., \& Moriarty-Schieven, G. H. 2004, ApJ, 601,962

Shu, F. H., Adams, F. C., \& Lizano, S 1987, ARA\&A, 14, 587

Shu, F. H., Shang, S., \& Lee, T. 1996, Science, 271, 1545

Sohn, J., Lee, C. W., Park, Y.-S., Lee, H. M., Myers, P. C., \& Lee, Y. 2007, ApJ, 664, 928

Stahler, S. W. 1988, ApJ, 332, 804

Stamatellos, D., Whitworth, A. P., Boyd, D. F. A., \& Goodwin, S. P. 2005, A\&A, 439,159

Stognienko, R., Henning, T. H., \& Ossenkopf, V. 1995, A\&A, 296, 797

Terebey, S., \& Fich, M. 1986, ApJ, 309, L73

Terebey, S., Fich, M., Noriega-Crespo, A., Padgett, D. L., \& Sullivan, A. 2005, in Proc. Protostars \& Planets V, 8568

Terebey, S., Shu, F. H., \& Cassen, P. 1984, ApJ, 286, 529

Terebey, S., Van Buren, D., Brundage, M., \& Hancock, T. 2006, ApJ, 637, 81

Testi, L., \& Sargent, A. I. 1998, ApJ, 508, 91

Tobin, J. J., Hartmann, L., Calvet, N., \& D’Alessio, P. 2008, ApJ, 679, 1364

Torres, R. M., Loinard, L., Mioduszewski, A. J., \& Rodriguez, L. F. 2007, ApJ, 671,1813

Weingartner, J. C., \& Draine, B. T. 2001, ApJ, 548, 296

Werner, M. W., et al. 2004, ApJS, 154, 1

White, R. J., \& Hillenbrand, L. A. 2004, ApJ, 616, 998

Whitney, B.A., Wood, K., Bjorkman, J. E., \& Wolff, M. 2003, ApJ, 591 , 1049

Wood, D. O. S., Myers, P. C., \& Daugherty, D. A. 1994, ApJS, 95, 457

Young, C. H., et al. 2004, ApJS, 154, 396 